\title{
Star counts in the Galaxy
}

\section{Simulating from very deep to very shallow photometric surveys with the TRILEGAL code ${ }^{\star}$}

\author{
L. Girardi ${ }^{1}$, M. A. T. Groenewegen ${ }^{2}$, E. Hatziminaoglou ${ }^{3,4}$, and L. da Costa $^{3}$ \\ 1 Osservatorio Astronomico di Trieste, INAF, via Tiepolo 11, 34131 Trieste, Italy \\ e-mail: lgirardi@ts.astro.it \\ 2 PACS ICC-team, Instituut voor Sterrenkunde, Celestijnenlaan 200B, 3001 Leuven, Belgium \\ 3 European Southern Observatory, Karl-Schwarzschild-Str. 2, 85740 Garching bei München, Germany \\ 4 Instituto de Astrofísica de Canarias, C/ vía Láctea s/n, 38200 La Laguna, Spain
}

Received 10 November 2004 / Accepted 15 March 2005

\begin{abstract}
We describe TRILEGAL, a new populations synthesis code for simulating the stellar photometry of any Galaxy field. The code attempts to improve upon several technical aspects of star count models, by: dealing with very complete input libraries of evolutionary tracks; using a stellar spectral library to simulate the photometry in virtually any broad-band system; being very versatile allowing easy changes in the input libraries and in the description of all of its ingredients - like the star formation rate, age-metallicity relation, initial mass function, and geometry of Galaxy components. In a previous paper (Groenewegen et al. 2002, Paper I), the code was first applied to describe the very deep star counts of the CDFS stellar catalogue. Here, we briefly describe its initial calibration using EIS-deep and DMS star counts, which, as we show, are adequate samples to probe both the halo and the disc components of largest scale heights (oldest ages). We then present the changes in the calibration that were necessary to cope with some improvements in the model input data, and the use of more extensive photometry datasets: now the code is shown to successfully simulate also the relatively shallower 2MASS catalogue, which probes mostly the disc at intermediate ages, and the immediate solar neighbourhood as sampled by Hipparcos - in particular its absolute magnitude versus colour diagram -, which contains a somewhat larger fraction of younger stars than deeper surveys. Remarkably, the same model calibration can reproduce well the star counts in all the above-mentioned data sets, that span from the very deep magnitudes of CDFS $(16<R<23)$ to the very shallow ones of Hipparcos $(V<8)$. Significant deviations (above 50 percent in number counts) are found just for fields close to the Galactic Center (since no bulge component was included) and Plane, and for a single set of South Galactic Pole data. The TRILEGAL code is ready to use for the variety of wide-angle surveys in the optical/infrared that will become available in the coming years.
\end{abstract}

Key words. stars: luminosity function, mass function - stars: Hertzsprung-Russell (HR) and C-M diagrams - surveys Galaxy: stellar content - Galaxy: structure

\section{Introduction}

The number counts of Galactic stars in a given bin of apparent magnitude $\left[m_{\lambda}, m_{\lambda}+\mathrm{d} m_{\lambda}\right]$ - where $\lambda$ stands for a passband and towards an element of galactic coordinates $(\ell, b)$ and solid angle $\mathrm{d} \Omega$, is given by the fundamental equation of stellar statistics (see Bahcall 1986, for a review)

$N\left(m_{\lambda}, \ell, b\right)=\mathrm{d} m_{\lambda} \int_{0}^{\infty} \mathrm{d} r r^{2} \rho(\boldsymbol{r}) \phi\left(M_{\lambda}, \boldsymbol{r}\right) \mathrm{d} \Omega$

where $r$ is the line-of-sight distance, and $\rho(\boldsymbol{r})$ is the stellar density as a function of the position $\boldsymbol{r}=(\ell, b, r) . r$, when measured in parsecs, is related to the absolute and apparent

* Appendix A is only available in electronic form at http://www. edpsciences.org magnitudes $M_{0, \lambda}$ and $m_{\lambda}$, and to the interstellar absorption $A_{\lambda}$, by

$M_{0, \lambda}=m_{\lambda}-5 \log r-A_{\lambda}(r)+5$.

$\phi\left(M_{0, \lambda}, \boldsymbol{r}\right)$ is the intrinsic distribution of stellar absolute magnitudes, i.e. the intrinsic luminosity function (LF) of the stars considered at $\boldsymbol{r}$.

To describe the stellar densities $\rho(\boldsymbol{r})$ for the largest possible volume, and to a lesser extent also $\phi\left(M_{\lambda}, \boldsymbol{r}\right)$, is the ultimate task of the so-called Galaxy star count models. To achieve these goals, the usual way is to assume the functional forms of $\rho$ and $\phi$, and then compare the results of Eq. (1) to observed number counts in several Galaxy fields. A number of assumptions help in simplifying the task. The first one is to recognize that the 
Galaxy can be separated in a few distinct components, such as the disc, halo, and bulge:

$\rho=\rho_{\mathrm{d}}+\rho_{\mathrm{h}}+\rho_{\mathrm{b}}$,

each one of these components having a simple expression for their density. The second one is to assume an intrinsic LF $\phi$ which is virtually independent of $\boldsymbol{r}$, i.e. $\phi\left(M_{\lambda}, \boldsymbol{r}\right)=\phi\left(M_{\lambda}\right)$, for each component.

The recipes for $\phi(M)$ can be of two types. Either one (1) assumes an empirical $\phi(M)$, derived from e.g. star counts in globular clusters or in the Solar Neighbourhood; or (2) assumes a theoretical $\phi(M)$, derived from a set of evolutionary tracks together with suitable distributions of stellar masses, ages, and metallicities.

Option (1) was the preferred one in the past, and the one adopted in some of the most successful Galaxy models. Despite their success in reproducing several sorts of data, it is not difficult to find points of inconsistency in many of such models. A common approximation, for instance, has been that disc stars of different absolute magnitude present different scale heights (and hence $\rho_{\mathrm{d}}(\boldsymbol{r})$ ): Bahcall \& Soneira (1980, 1984; and many authors later on) assigned the scale height of $325 \mathrm{pc}$ for $M_{V}>5.1$, of $90 \mathrm{pc}$ for $M_{V}<2.3$ dwarfs, and linearly interpolated in between. This separation was interpreted as a coarse separation into "young" and "old" populations. Red giants instead were assigned 250 pc. Gilmore \& Reid (1983) adopt similar approximations for main sequence stars. Méndez \& van Altena $(1996,1998)$ do the same, and moreover assume a unique scale height for all evolved stars (subgiants, giants, and white dwarfs). From the point of view of stellar and population synthesis theories, these approximations are clearly not justified, for a series of reasons: (a) Most coeval stellar populations contain both red and blue stars characterized by initial mass values which are, at least for the most luminous objects, very much the same; it is then very unlikely that the spatial distribution of these red and blue stars could be different. (b) Similarly, young stellar populations contain both bright and faint main sequence stars, whose relative scale heights cannot change that dramatically in time-scales of less than one Gyr. (c) Moreover, it is remarkable that population synthesis theory indicate that in star-forming galaxy components (e.g. in the thin disc), red giants are relatively young - most having less than say 2 Gyr (see Girardi \& Salaris 2001) - and not old objects; applying the largest scale heights to all giants is then simply wrong.

Although this sort of inconsistency is not inherent to method (1), they are completely removed by the use of method (2). In the latter, at any $\boldsymbol{r}$ the relative numbers of stars with different colours and absolute magnitude strictly obey the constraints settled by stellar evolution and population synthesis theories; hence, $\rho(\boldsymbol{r})$ cannot be arbitrarily changed as a function of absolute magnitude. On the other hand, method (2) allows $\rho(\boldsymbol{r})$ to be easily expressed as a function of other stellar parameters, such as age and metallicity - something not possible with method (1) where individual stellar ages and metallicities are not available - then allowing the simulation of important effects like metallicity gradients, scale lengths increasing with age, etc. This turns out to be a significant advantage of method (2) over (1).
Models that follow method (2) may be put under the generic name of "population synthesis Galaxy star count models", and have been developed in the last decades by e.g. Robin \& Crézé (1986), Haywood (1994), Ng et al. (1995), Castellani et al. (2002), and Robin et al. (2003). These works benefit from the releases of extended databases of stellar evolutionary tracks to predict the properties of stars of given mass, age, and metallicity. Some assumptions then are necessary to give the distributions of these stellar parameters. Such distributions may be derived, for instance, starting from an initial mass function (IMF), an age-metallicity relation (AMR), and a law for the star formation rate (SFR) as a function of Galaxy age.

In the present paper, we will describe a Galaxy model developed according to the population synthesis approach, taking particular care in the consistency among the different sources of input data. It has been developed with a primary task in head, which is, essentially: to be capable of simulating the same sort of data that will be released by some major campaigns of widefield photometry conducted these years. Of primary importance in this context, are the several parts of the ESO Imaging Survey (EIS, Renzini \& da Costa 1997), the Two Micron All Sky Survey (2MASS, Cutri et al. 2003), and the Sloan Digital Sky Survey (SDSS, York et al. 2000). Present and future data from HST deep fields, VIMOS, VISTA, UKIDSS, GAIA, might be considered as well. Moreover, our model should also be able to take advantage of the extraordinary constraints provided by the astrometric mission Hipparcos (Perryman et al. 1997). Of course, a program which meets these aims can be applied to any other sort of wide field data as well.

Before proceeding, let us briefly summarize our primary objectives and how these translate into technical requirements.

First of all, our primary goal is to simulate the expected star counts in several passband systems, such as those used by Hipparcos, EIS, 2MASS, SDSS, etc. For doing so, we should be able to consistently predict the stellar photometry in a lot of different photometric systems. The way out to this problem has been settled in a previous work (Girardi et al. 2002), that describes a quite general method for performing synthetic photometry and deriving bolometric corrections from an extended library of stellar spectra. Such tables are now routinely produced for any new system that we want to compare the models with.

The second requirement is of being able to simulate both very shallow - but of excellent quality - photometric data samples as Hipparcos (Perryman et al. 1997), and very deep ones such as the EIS Deep Public Survey (e.g. Paper I). The former case implies that we include all important evolutionary sequences, such as most of the main sequence, and giants both in the red giant branch (RGB) and red clump, which make the bulk of the Hipparcos colour-magnitude diagram (CMD). In the latter case, we should also include an extended lower main sequence, reaching down to visual absolute magnitudes as faint as $M_{V} \sim 30$, which corresponds to stellar masses of $\sim 0.1 M_{\odot}$. Moreover, old white dwarfs start to become frequent at such faint magnitudes as well. Therefore, the libraries of stellar data should be extended to the intervals of very low masses, and to very old white dwarfs. 


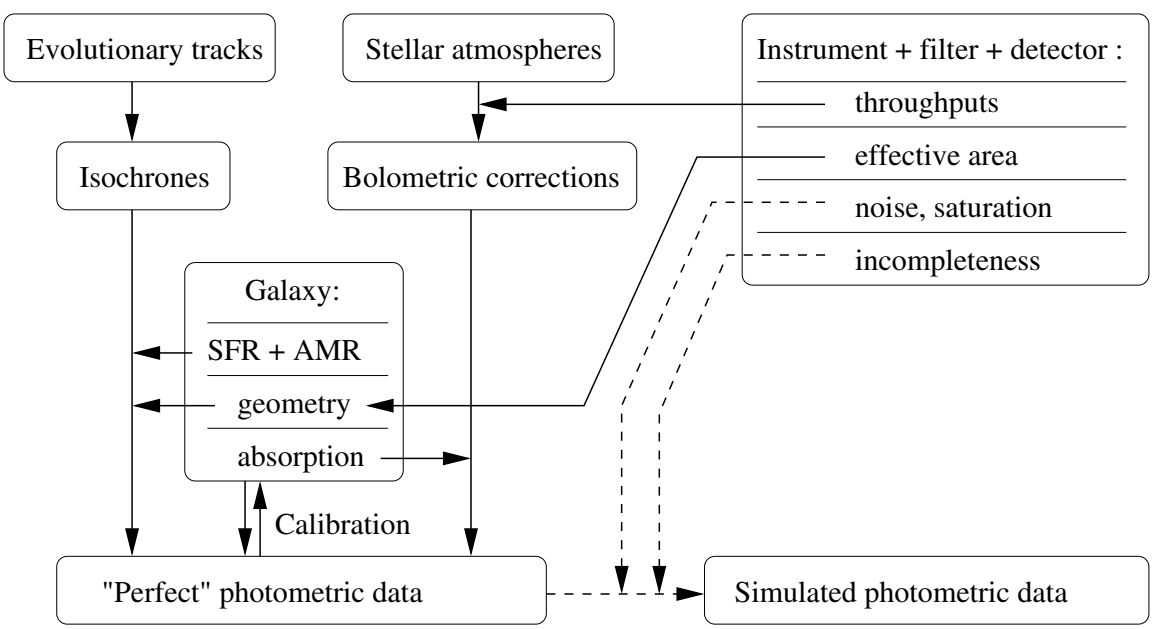

Fig. 1. A general scheme of our codes. The continuous arrows refer to steps which are performed inside the TRILEGAL main code and subroutines; they lead to the simulation of perfect (i.e. without errors) photometric data. The dashed lines refer to some optional steps, usually performed with external scripts, like e.g. those mentioned in Paper I, to generate catalogues with errors.

It is also clear that these requirements imply, necessarily, that we opt for the population synthesis approach. In fact, there is little hope that we could collect empirical data for such a variety of stars, in the several photometric systems involved, and with good enough statistics that the intrinsic CMDs could be constructed with reliability. Theoretical data, instead, is available for all of our purposes, as will be shown in what follows. Such theoretical data is also routinely submitted to stringent tests against photometric data, such as the Hipparcos CMD, star clusters, eclipsing binaries, red giants with measured diameters, etc. In general, the errors detected in the models amount to less than a few tenths of a magnitude, and just for some particular stars and/or passbands. Certainly, the time is ripe for completely relying in theoretical data in Galaxy star count models.

The plan of this paper is as follows: Sects. 2 and 3 detail the code and its input data, respectively. Section 4 describes its initial calibration as performed for Groenewegen et al.'s (2002) work, based mostly on EIS and DMS data. Section 5 details and presents the fine-tuning of the initial calibration, including additional comparisons with 2MASS and Hipparcos data. Section 6 draws a few final comments and summarizes the main results of the present paper.

\section{The code}

We describe here all the necessary input for computing a Galactic Model. Normally, this means simulating the photometric properties of stars located towards a given direction $(\ell, b)$. This task is performed by the newly developed code TRILEGAL, which stands for TRIdimensional modeL of thE GALaxy ${ }^{1}$.

The code is written in $\mathrm{C}$ language. Its core is made of a few subroutines that efficiently interpolate and search for stars of given mass, age, or metallicity, inside a database of stellar evolutionary tracks. They deal with all the intrinsic properties of stars - luminosity, effective temperature, mass, metallicity, etc. These subroutines, developed in Girardi (1997), have so far been used in a series of works dealing from the construction of theoretical isochrones (e.g. Girardi et al. 2000;

\footnotetext{
1 TRILEGAL is also a word commonly used to say "very nice" in southern Brazil.
}

Salasnich et al. 2000) to the simulations of synthetic CMDs for nearby galaxies (e.g. Girardi et al. 1998; Girardi 1999; Girardi \& Salaris 2001; Marigo et al. 2003). Another set of routines, more recently developed, deal with all aspects related with synthetic photometry, i.e. the conversion between intrinsic stellar properties and observable magnitudes. They rely on the same simple formalism described in Girardi et al. (2002).

\subsection{Scheme}

A general scheme of the code is provided in Fig.1. It makes use of 4 main elements: a library of theoretical evolutionary tracks, a library of synthetic spectra, some parameters of the detection system, and the detailed description of the Galaxy components. The libraries of evolutionary tracks and spectra can be pre-processed in the form of theoretical isochrones and tables of bolometric corrections, so as to reduce the number of redundant operations during a simulation. These are to be considered as "fixed input", but can be easily changed so as to consider alternative sets of data.

The instrumental setup specifies, among others: (1) the set of filters+detector+telescope throughputs in which the observations are performed; any change of them implies the recalculation of the bolometric correction tables; (2) the effective sky area to be simulated; the number of simulated stars scales with this quantity.

The several Galactic components (halo, thin and thick disc, bulge, etc.) are specified by their initial distributions of stellar ages and metallicities (SFR and AMR), masses (IMF), space densities, and interstellar absorption. This is done separately for each component. The space densities are in the form of simple expressions containing a few modifiable parameters, to be specified in Sect. 3 below.

\subsection{The simulation and output}

A run of TRILEGAL is formally a Monte Carlo simulation in which stars are generated according to the probability distributions already described. Equation (1) is used for predicting the number of expected stars in each bin of distance modulus. For each simulated star, the SFR, AMR and IMF are used to single 
out the stellar age, metallicity, and mass. Finally its absolute photometry is derived via interpolation in the grids of evolutionary tracks (or isochrones), and converted to the apparent magnitudes using the suitable values of bolometric corrections, distance modulus and extinction.

During the simulations, a lot of different stellar parameters can be kept in memory and printed out: initial and current mass, age, metallicity, surface chemical composition and gravity, luminosity, effective temperature, core mass, etc. In the case of thermally-pulsing (TP-) AGB stars, this information is also used to simulate the pulse cycle variations (see Marigo et al. 2003).

The calculation initially produces a "perfect photometric catalogue", which perfectly reflect the input probability distributions but for the Poisson noise. This catalogue can be later degraded by using the known photometric errors, such as photon noise, saturation and incompleteness for a given instrumental setup. This second task does not belong to TRILEGAL, but is performed by separated subroutines, like for instance the ones described by Paper I.

\section{The input datasets}

There are essentially 5 different input datasets in TRILEGAL:

1. tables of stellar evolutionary tracks, that give their basic properties (bolometric magnitude $M_{\mathrm{bol}}$, effective temperature $T_{\text {eff }}$, surface gravity $g$, core mass, surface chemical composition, etc.) as a function of initial mass $M_{\mathrm{i}}$, stellar age $\tau$, and metallicity $Z$;

2. tables of bolometric corrections $B C_{\lambda_{i}}$ for the several filter pass-bands $\lambda_{i}$, as a function of $T_{\text {eff }}, \log g$, and $[\mathrm{M} / \mathrm{H}]$, as well as the relative absorption in the several passbands with respect to $V, A_{\lambda_{i}} / A_{V}$;

3. the IMF $\phi_{m}$;

4. the star formation rate as a function of age, $\psi(t)$, and agemetallicity relation, $Z(t)$, for the different Galaxy components;

5. the geometry of the Galaxy components, i.e. the stellar density $\rho(\boldsymbol{r})$ and differential $V$-band extinction $\mathrm{d} A_{V}(\boldsymbol{r})$ as a function of position $\boldsymbol{r}$.

They will be discussed separately in the following.

\subsection{Evolutionary tracks}

Based on our previous work on simulations of synthetic CMDs for Local Group galaxies, we have assembled a large, quite complete, and as far as possible homogeneous - in terms of their input physics - database of stellar tracks. They are illustrated in the HR diagram of Fig. 2:

1) for masses between $0.2 M_{\odot}$ and $7 M_{\odot}$, we use the tracks from Girardi et al. (2000), that range from the zeroage main sequence (ZAMS) up to either the end of the TP-AGB, or to an age of $25 \mathrm{Gyr}$ for the lowest-mass stars. Metallicities are comprised between $Z=0.0004$ $([\mathrm{M} / \mathrm{H}]=-1.7)$ and $0.03([\mathrm{M} / \mathrm{H}]=+0.2)$. Another set with

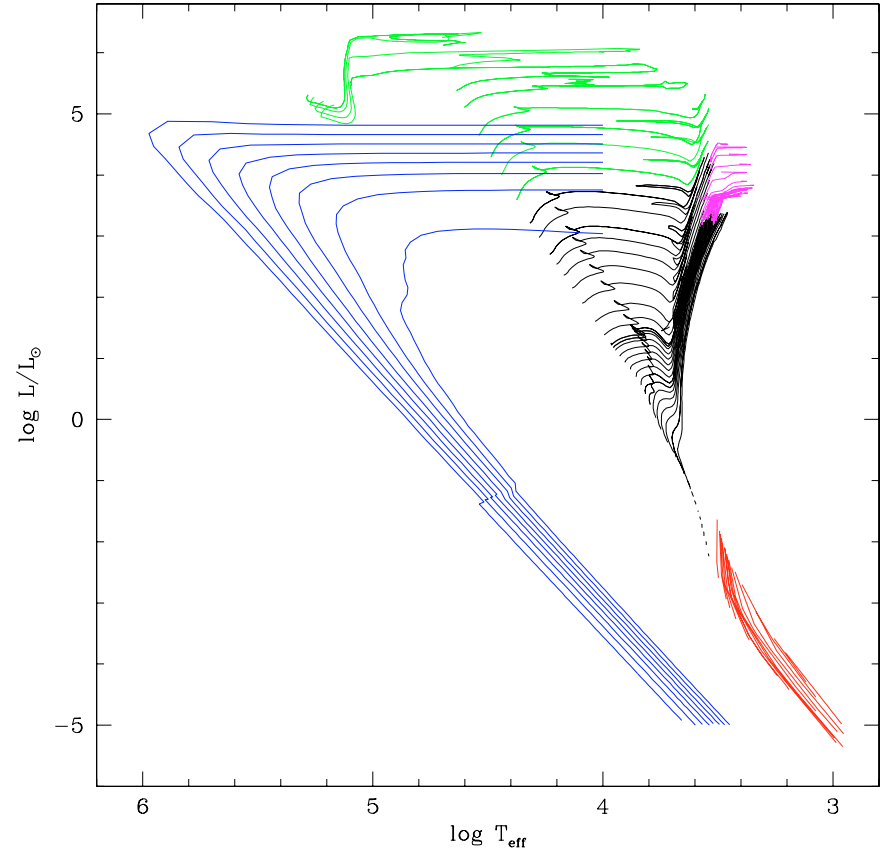

Fig. 2. HR diagram containing all tracks assembled for the solar metallicity. Our database contains similar data for 6 other values of metallicity. In the electronic version of this paper, tracks from different sources are marked with different colours: Girardi et al. (2000, black) for most evolutionary phases of low- and intermediate-mass stars, complemented with the TP-AGB phase from Marigo et al. (2003, and in preparation, magenta), massive stars from Bertelli et al. (1994, green), very-low mass stars and brown dwarfs from Chabrier et al. (2000, red), post-AGB and PNe nuclei from Vassiliadis \& Wood (1994) complemented with WD cooling sequences from Benvenuto \& Althaus (1999, both in blue).

$Z=0.0001([\mathrm{M} / \mathrm{H}]=-2.3)$ and computed with the same input physics (Girardi, unpublished) is included.

The TP-AGB evolution included in these tracks is estimated from a simplified synthetic evolutionary code (cf. Girardi \& Bertelli 1998, case of Eqs. (17) plus (20)). Although this TP-AGB evolution is very approximated, it provides a reasonable initial-final mass relation (see Fig. 2 in Girardi \& Bertelli 1998), and hence reasonable masses for the white dwarfs to be considered below. The maximum mass of WDs attained is about $1.2 M_{\odot}$ for the lowest metallicities, and 0.9 for the highest. On 2002, we replaced these simplified TP-AGB tracks for more detailed ones computed by Marigo et al. (2003; and in preparation), which are based on the new formulation for molecular opacities by Marigo (2002);

2) for masses lower than $0.2 M_{\odot}$, and down to $0.01 M_{\odot}$, we include the models for very-low mass stars and brown dwarfs with dusty atmospheres from Chabrier et al. (2000). This provides us with a main sequence (MS) going down to luminosities as faint as $\log \left(L / L_{\odot}\right)=-5$, and to effective temperatures as cold as $916 \mathrm{~K}$. For $M<0.2 M_{\odot}$, Chabrier et al. (2000) tracks exist only for solar metallicity, which are hence adopted for all metallicities in our models. It is also worth remarking that Chabrier et al. tracks evolve on time-scales of some Gyr, and include a significant fraction of the pre-MS evolution; 
3) post-AGB and white dwarfs tracks of a suitable mass are attached at the end of the TP-AGB phase of all stars of initial mass between 0.6 and $5 M_{\odot}$. We use the post-AGB and PNe nuclei tracks from Vassiliadis \& Wood (1994) down to $\log L / L_{\odot} \simeq-1.5$. We then shift to the WD cooling tracks of Benvenuto \& Althaus (1999) with CO cores, total mass ranging from 0.5 to $1.1 M_{\odot}$, a $10^{-6} M_{\odot}$ envelope layer of hydrogen, and non-zero metallicity, plus their $1.2 M_{\odot}$ model of zero-metallicity. Unfortunately, these tracks end at $\log \left(L / L_{\odot}\right)=-5$, which implies maximum WD ages of about 10 Gyr. To overcome this problem, we have artificially extended the tracks up to ages of 15 Gyr. Notice that no track is available for WDs between 1.2 and $1.4 M_{\odot}$ (the Chandrashekhar mass). This is not a problem at all, since from our AGB tracks we predict no WDs within this range of masses;

4) for masses higher than $7 M_{\odot}$ we adopt the same tracks as in Bertelli et al. (1994) isochrones, but for $Z=0.0001$ and $Z=0.001$ where more recent models (Girardi et al. 1996 and 2003, respectively) are used.

We interpolate among all these tracks in order to derive stars with intermediate values of mass, age, and metallicity. All interpolations are performed between points of equivalent evolutionary status, as usual in the codes for generating isochrones (e.g. Bertelli et al. 1994; Girardi et al. 2000). Linear interpolations are adopted, with $\log m, \log t$, and $[\mathrm{M} / \mathrm{H}]$ being the independent variables.

The complete set of stellar models for solar metallicity is plotted in the HR diagram of Fig. 2. Similar grids of tracks are available also for metallicities $Z=0.0001,0.0004,0.001$, $0.004,0.008$ and 0.03 (limited to $M \leq 7 M_{\odot}$ in the latter case).

Finally, we remark that the present stellar database corresponds to the "basic set" of isochrones as mentioned by Girardi et al. (2002) and available at the web page http://pleiadi.pd.astro.it/isoc_photsys.00.html, but for three important improvements: the inclusion of post-AGB and white dwarf cooling tracks, the extension of very-low mass stars and brown dwarfs down to $0.01 M_{\odot}$, and the improved prescriptions for the TP-AGB phase.

\subsection{Tables of bolometric corrections and absorption coefficients}

Once a star of $\left(L, T_{\text {eff }},[\mathrm{M} / \mathrm{H}]\right)$ is selected by the code, its bolometric luminosity $M_{\mathrm{bol}}=-2.5 \log L+M_{\mathrm{bol} \odot}$ is converted into absolute magnitudes by means of $M_{\lambda}=M_{\mathrm{bol}}-B C_{\lambda}$. The bolometric corrections $B C_{\lambda}$ are derived from a large database of synthetic and empirical spectra, according to the synthetic photometry procedure throughly descrived in Girardi et al. (2002), Importantly, this allows the application to a very wide set of photometric systems, provided that we deal with

- intermediate- to broad-band filter sets (otherwise errors in synthetic colours become significant);

- VEGA, AB, or ST magnitudes, or systems in which some of the photometric standard stars are also well-measured spectrophotometric standards.
Regarding the spectral library in use, it is essentially the same one described in Girardi et al. (2002), but now complemented with white dwarf spectra. Namely, the sources of spectra are:

1. for most stars, the "non-overshooting" set of models computed by Castelli et al. (1997) with the Kurucz (1993) ATLAS9 code (see also Bessell et al. 1998). This set cov-

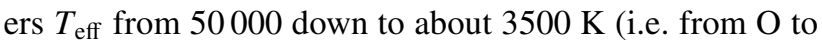
early-M spectral types), and metallicities $[\mathrm{M} / \mathrm{H}]$ from +0.5 to -2.5 ;

2. blackbody spectra for stars with $T_{\mathrm{eff}}>50000 \mathrm{~K}$;

3. empirical spectra for M giants (Fluks et al. 1994);

4. "BDdusty1999" spectra for all dwarfs cooler than $3900 \mathrm{~K}$, and down to $500 \mathrm{~K}$ (Allard et al. 2000);

5. synthetic DA white dwarf atmospheres from Finley et al. (1997) and Homeier et al. (1998), for $T_{\text {eff }}$ between 100000 and $5000 \mathrm{~K}$.

In addition to the $\mathrm{BC}$ values for stars of any $\left(T_{\mathrm{eff}}, \log g,[\mathrm{M} / \mathrm{H}]\right)$, we also compute, for any photometric system, the ratio between the absorption coefficient in each filter, and the total absorption in the $V$ band, $A_{\lambda} / A_{V}$. As it is well known (see e.g. Grebel \& Roberts 1995; Girardi et al. 2004), this ratio is not strictly constant but depends on the spectral type of the star and the extinction curve under consideration.

In the present work we compute the $A_{\lambda} / A_{V}$ ratio for a G2V star (the Sun) subject to mild absorption $\left(A_{V}<0.5 \mathrm{mag}\right)$ and following the Cardelli et al. (1989) absorption curve with $R_{V}=3.1$. The derived $A_{\lambda} / A_{V}$ quantities $^{2}$ are then applied to stars of all spectral types and reddening values, although, formally, they are adequate only for low-reddening G2V stars in the case $R_{V}=3.1$. This approach is adopted just for the sake of simplicity. In alternative, it is very easy to implement a more accurate approach to the problem, which will be followed in future applications.

\subsection{The initial mass function}

The IMF $\phi_{m}$ is a crucial ingredient because it determines the relative numbers of very-low mass stars, that may dominate star counts at visual magnitudes fainter than $\sim 22$. We have introduced the IMF in a very flexible way, so that it that can be easily changed. In order to be able to use star formation rates in units of $M_{\odot} / y r$, our default IMF normalization is for a total mass equal to 1 , i.e.

$\int_{0}^{\infty} m \phi_{m} \mathrm{~d} m=1 M_{\odot}$.

Our default IMF is a Chabrier (2001) log-normal function,

$\phi_{m} \propto m \exp \left[-\frac{\left(\log m-\log m_{0}\right)^{2}}{2 \sigma^{2}}\right]$,

whose parameters are a characteristic mass, $m_{0}=0.1 M_{\odot}$, and dispersion, $\sigma=0.627$.

Other commonly-used IMFs, like segmented power-laws (Salpeter 1955; Kroupa 2001) and Larson's (1986) exponential form, are also included in the code.

${ }^{2}$ Extinction coefficients for the Johnson-Cousins-Glass and SDSS photometric systems are tabulated in Girardi et al. (2004). 
The IMF as given above refers to the mass distribution of single stars. Additionally to them, it is very easy to simulate non-interacting binaries in our simulations. When so required, we adopt the same prescription as in Barmina et al. (2002): for each primary star of mass $m_{1}$, there is a probability $f_{\mathrm{b}}$ that it contains a secondary, whose mass $m_{2}$ is given by a flat distribution of mass ratios comprised in the interval $\left[b_{\mathrm{b}}, 1\right]$. This prescription is particularly useful for simulating the binary sequences which are often evident in CMDs of open clusters. Typical values of $f_{\mathrm{b}}$ and $b_{\mathrm{b}}$ are 0.3 and 0.7 , respectively, which we adopt as a default.

\subsection{Star formation rates and age-metallicity relations}

Each Galaxy component is made of combination of stellar populations of varying age and metallicity. In our code, their distribution is completely specified by the functions SFR, $\psi(t)$, and AMR, $Z(t)$. Both are given in a single input file containing, for each age value,

- the SFR $\psi(t)$ (in units proportional to $M_{\odot} / \mathrm{yr}$ );

- the mass fraction of metals $Z$;

- the logarithmic dispersion of $Z, \sigma(\log Z)$.

Whenever necessary, $Z$ is converted into the logarithmic metal and iron contents by means of the approximate relations

$[\mathrm{M} / \mathrm{H}]=\log \left(Z / Z_{\odot}\right)$,

$[\mathrm{Fe} / \mathrm{H}]=\log \left(Z / Z_{\odot}\right)-[\alpha / \mathrm{Fe}]$,

where $[\alpha / \mathrm{Fe}]$ is the degree of enhancement of $\alpha$ elements with respect to scaled-solar compositions, and $Z_{\odot}=0.019$ as in Girardi et al. (2000). These relations provide $[\mathrm{M} / \mathrm{H}]$ and $[\mathrm{Fe} / \mathrm{H}]$ values accurate to within $\sim 0.03 \mathrm{dex}$, which is good enough for our purposes.

For this paper, $\alpha$ enhancement is taken into consideration for the low-metallicity Galaxy components (halo and old disc). In these cases, we can safely convert a given $[\mathrm{Fe} / \mathrm{H}]$ into $Z$ by means of Eq. (5), and associate to that $Z$ the evolutionary tracks computed with scaled-solar compositions using the relations provided by Salaris et al. (1993). For metallicities higher than about half solar, this approximation is no longer valid and it is preferable to use tracks specifically computed for $\alpha$-enhanced composition (see Salaris \& Weiss 1998; VandenBerg et al. 2000; Salasnich et al. 2000).

The SFR can only be considered well-known for the old Galactic components. In fact, for ages close to $12 \mathrm{Gyr}$, a change of age of 1 Gyr causes just small changes in the stellar luminosity function, and hence has a minor impact on the simulated star counts. For the disc components, the SFR is less constrained. Anyway, the Galactic model is also relatively insensitive to the disc SFR, at least as long as we are not sampling regions at low Galactic latitude and/or the Solar vicinity (as will be shown later). In general, even more important than the SFR is the choice of the AMR $Z(t)$ and its dispersion, that may change the position of simulated stars in colour-colour diagrams, and cause a significant colour dispersion.
After these considerations, it is convenient to specify what are to be considered "default" SFR and AMR - i.e. those used in Paper I, and partially also in this work:

- the SFR was assumed to be constant over the last $11 \mathrm{Gyr}$ for the disc, and constant between 12 and 13 Gyr for the halo;

- the AMR for the disc was taken from Rocha-Pinto et al. (2000). $[\mathrm{Fe} / \mathrm{H}]$ values are converted into the metal content $Z$ by means of a relation that allows for $\alpha$-enhancement at decreasing $[\mathrm{Fe} / \mathrm{H}]$, as suggested by Fuhrmann (1998) data. At any age, $[\mathrm{Fe} / \mathrm{H}]$ was assumed to have a $1 \sigma$ dispersion of 0.2 dex;

- the metallicity of the halo stars was assumed be $Z=$ 0.0095 , with a dispersion of 1.0 dex. This was based on an observed $[\mathrm{Fe} / \mathrm{H}]$ value of $-1.6 \pm 1.0$ (Henry \& Worthey 1999), allowing for an $\alpha$-enhancement of 0.3 dex.

\subsection{The intrinsic colour-magnitude diagrams and luminosity functions}

At this point, having described the default SFR, AMR, IMF, and libraries of evolutionary tracks and spectra, it is useful to open a parenthesis and illustrate the intrinsic CMDs and LFs that we derive from these ingredients. Notice that these intrinsic data are the only stellar ingredients that enter in the old "empirical $\phi(M)$ approach" mentioned in Sect. 1.

In Fig. 3 we show, for both disc and halo, the $M_{V}$ vs. $B-V$ diagram that follow from our choices, together with the intrinsic distributions of $M_{V}$ and $B-V$ values.

Several aspects of this figure are remarkable:

- it is evident that we have a very complete sampling of the possible evolutionary stages of the stellar populations required in building a realistic Galactic model. Limiting to the bright part of the diagrams, we can notice the presence of the young disc main sequence, subgiants and giants, red clump and horizontal branch stars, that make most of the stars observed in shallow photometric surveys;

- the inclusion of white dwarfs, very low-mass stars and brown dwarfs allows us to simulate all faint stellar objects, including the extremely dim ones - as $\operatorname{dim}$ as $M_{V} \sim 44$. These faint objects are expected to appear in significant numbers in very deep surveys;

- one should keep in mind that we know, for each simulated star, many other physical parameters like the complete photometry, age, metallicity, surface gravity, etc., so that the same kind of plot could be constructed in a multitude of other ways and dissected by grouping stars in different parameter bins.

In summary, the present plots are an evindence of the significant advantages of an evolutionary synthesis tool, over the empirical approaches which were used in the past to construct similar data for Galaxy models.

Let us also briefly comment on the general aspect of the CMDs shown in Fig. 3: in the case of the disc population shown in the left panel, it is evident that the low-mass and brown dwarf models, taken from different sources, combine in a continuous 

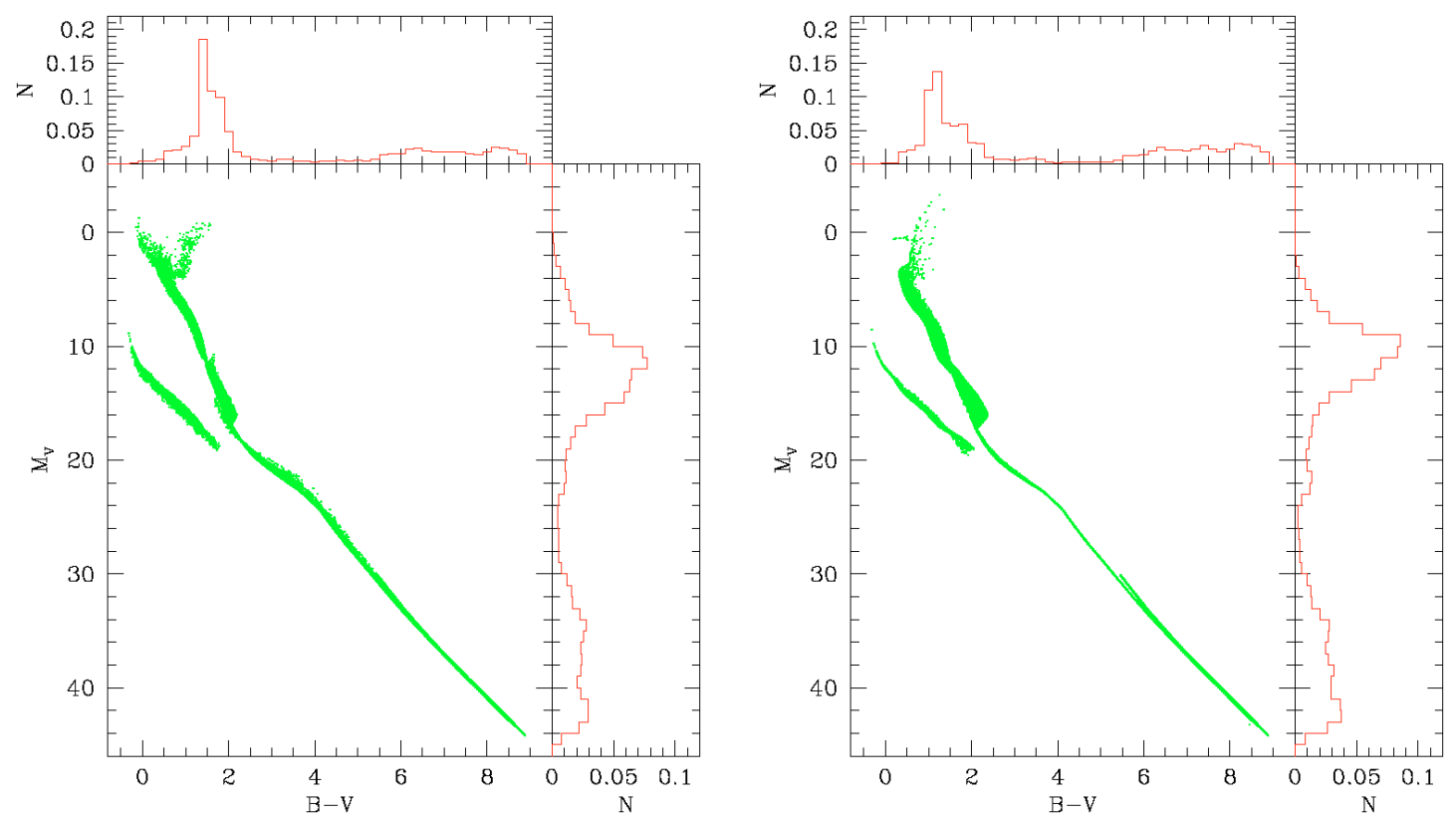

Fig. 3. Intrinsic $M_{V}$ vs. $B-V$ diagrams that follow from our default choices of SFR, AMR, IMF, evolutionary tracks and spectra, shown for both the disc (left) and halo (right). In both cases, the histograms to the right and top show the corresponding luminosity and colour functions, respectively. Each diagram contains about $10^{5}$ simulated objects.

and well-behaved way with the sequence drawn by more massive stellar models. There is just one abrupt change in the width of this main sequence, occurring at $M_{V} \sim 19$, that is caused by the fact that below this limit we rely on solar-metallicity models, whereas above it the metallicity dispersion is fully represented in the models. Exactly the same problem is present for the halo population shown in the right panel. Anyway, this seems a minor problem because - as will be shown in the following sections - these stars, although always present, do not make the main features of CMDs observed up to now. Also, this seems an acceptable price to pay for having an extremely complete intrinsic CMD.

Finally, we recall that star counts of dwarfs below $M_{V} \sim 6$ are affected just by the particular choice of IMF, whereas above this limit also the SFR and AMR play a major role.

For the sake of illustration, Fig. 4 shows the intrinsic $M_{K}$ vs. $J-K$ diagram. This looks very different from the former $B V$-diagram, and is particularly useful for the discussion of 2MASS data (see Sect. 5.3, and Marigo et al. 2003).

\subsection{Geometry of Galaxy components}

Five are the Galaxy components presently defined in TRILEGAL: the thin and thick discs, the halo, the bulge, and the disc extinction layer. There is also the possibility of simulating additional objects of known distance.

The thin disc: its density is assumed to decrease exponentially with the galactocentric radius projected onto the plane of the disc, $R$,

$\rho_{\mathrm{d}}=C_{\mathrm{d}} \exp \left(-R / h_{R}\right) f(z)$.

The vertical distribution $f(z)$ is either an exponential, $\exp \left(-|z| / h_{z}\right)$, or a squared hyperbolic secant function,

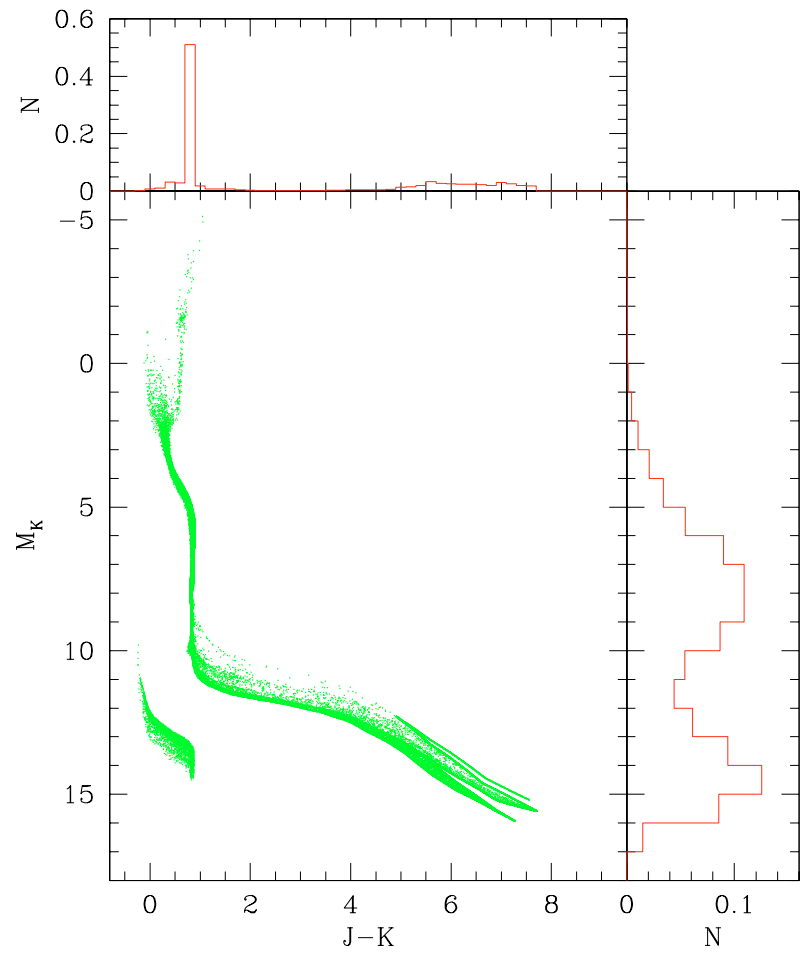

Fig. 4. The same as Fig. 3 but limited to the disc intrinsic $M_{K}$ vs. $J-K$ diagram.

$\operatorname{sech}^{2}\left(0.5 z / h_{z}\right)$. Importantly, the vertical scale heigth $h_{z}$ is assumed to increase with the stellar age $t$ according to the formula suggested by Rana \& Basu (1992):

$h_{\mathrm{d}}(t)=z_{0}\left(1+t / t_{0}\right)^{\alpha}$,

where $z_{0}, t_{0}$ and $\alpha$ are adjustable parameters. This means that stars are formed very close to the Galaxy Plane, with a scale 
heigth $z_{0}$, and later disperse vertically. For practical reasons, the code has to deal with a limited number of age intervals and scale heigths. Thus, the total age interval is divided into a number of $N_{\mathrm{d}}$ subintervals - at least 10 , but typically $100-$ and the stellar densities computed separately for them.

The normalization constant $C_{\mathrm{d}}$ is set so as to produce a given "total surface density of thin disc stars ever formed in the Solar Neighbourhood",

$\Sigma_{\mathrm{d}}(\odot)=\int_{0}^{t_{\mathrm{G}}} \psi_{\mathrm{d}}(t) \mathrm{d} t \int_{-\infty}^{+\infty} \rho_{\mathrm{d}}(\boldsymbol{r})_{R=R_{\odot}} \mathrm{d} z$

where $\psi_{\mathrm{d}}(t)$ is the SFR per unit disc area in the so-called Solar Cylinder $^{3}$, and $t_{\mathrm{G}}$ is the Galaxy age. So, the thin disc geometry is completely defined by the parameters $\Sigma_{\mathrm{d}}(\odot), h_{\mathrm{R}}, z_{0}, t_{0}$ and $\alpha$. Additionally, the parameter $N_{\mathrm{d}}$ may be adjusted to provide better accuracy at the cost of larger CPU times. In present applications we use $N_{\mathrm{d}}$ as large as 100 , which in fact represents an excellent accuracy.

The thick disc: similarly to the thin disc, it is described either by a double exponential or by an exponential times a sech ${ }^{2}$ function. However, the scale height is assumed to be independent of age. This because the thick disc is always described by predominantly old ( $t \geq 10 \mathrm{Gyr}$ ) populations.

So, just the parameters $\Sigma_{\mathrm{td}}(\odot), h_{R, \mathrm{td}}$, and $h_{z, \mathrm{td}}$, defined similarly to the thin disc ones, would suffice to define the thick disc. Needless to say, this galactic component can be incorporated in the formula for the thin disc, by assuming suitable scale heights $h_{z}$ at large ages in Eq. (7).

The halo: its density $\rho_{\mathrm{h}}$ is given by a de Vaucoulers (1959) $r^{1 / 4}$ law, deprojected according to Young (1976). Alternatively, an oblate spheroid (Gilmore 1984) can be assumed. The halo parameters are: the radial scale $r_{\mathrm{h}}$, the oblateness $b_{\mathrm{h}}$, and the local "total density of halo stars ever formed in the Solar Neighbourhood",

$\Omega_{\mathrm{h}}(\odot)=\rho_{\mathrm{h}}\left(\boldsymbol{r}_{\odot}\right) \int_{0}^{t_{\mathrm{G}}} \psi_{\mathrm{h}}(t) \mathrm{d} t$.

The bulge: although it is included in the code as a triaxial truncated spheroid, it has not yet been calibrated. For this reason no bulge component is included in any of the calculations presented in this paper.

The disc extinction layer: it is assumed to have an exponentially-decreasing density in the vertical direction, with a scale-height $h_{z}^{\text {dust }}$ (Parenago 1945; cf. Méndez \& van Altena 1998). The increase of $A_{V}$ with distance is proportional to this density. This geometric distribution is normalized in two possible ways: either adopting a local absorption density $A_{V}^{0}$, of about $0.75 \mathrm{mag} / \mathrm{kpc}$ (Lyngå 1982), or adopting a total absorption at infinity $A_{V}^{\infty}$ as given by Schlegel et al. (1998) maps.

\footnotetext{
3 The Solar Cylinder refers to a cylinder perpendicular to the Galaxy Plane, of small diameter and infinite height, centered on the Sun.
}

We set the second option to be the default one. We adopt $h_{z}^{\text {dust }}=110$ pc as Lyngå (1982).

Extinction is always specified in terms of $A_{V}$ ( $V$ in this case stands for the effective wavelength of Johnson's $V$-band, $5550 \AA$ ). The $A_{V}$ values found for individual stars are later converted to those in the several pass-bands, $A_{\lambda}$, using the $A_{\lambda} / A_{V}$ ratios previously tabulated (Sect. 3.2).

Additional objects: they can be inserted at known distance, and optionally assuming a known foreground absorption. This alternative is useful for including objects such as star clusters or nearby satellite galaxies in the simulations. Of course, specifying the SFR and AMR is also necessary, which for star clusters is limited to a single age and metallicity value. The object total mass is again expressed in units of total numbers of stars ever born in that area of the sky.

This option was recently used by Marigo et al. (2003) to simulate the 2MASS data towards the Large Magellanic Cloud (LMC), and by Carraro et al. (2002) to simulate the field of the open cluster NGC 2158. Open cluster simulations in EIS fields will also be the subject of an upcoming paper (Hatziminaoglou et al., in preparation).

\subsection{Additional parameters}

We also have "pointing parameters", that specify the region of the Galaxy sampled during a simulation. Two main modes are presently allowed: either (1) simulations of a projected (conic) region of the sky, that requires the specification of the central Galactic coordinates $(\ell, b)$ and total sky area; or (2) a volumelimited sample centered on the Sun and complete up to a specified maximum distance.

In both of the above cases, by specifying a given limiting magnitude in any of the available filters, we avoid generating too many faint stars in the output.

Another input parameter is the resolution in magnitudes, $\Delta m$. It represents the largest sub-step for the numerical integration of Eq. (1). Any detail of the Galaxy geometry that is caused by a depth structure (in distance modulus) smaller than this resolution, or by a LF structure finer than it, will be lost. A resolution of $0.1 \mathrm{mag}$ is adequate for the purpose of this paper.

The Sun's position with respect to the Galactic Plane is specified by the galactocentric radius and height on disc.

\section{The initial calibration}

The initial calibration is described in Paper I. The most important points, relevant for the present paper, are repeated here. That paper describes, amongst other things, the first application of the TRILEGAL code. The initial calibration is derived from the six fields at high galactic latitude covered by the "Deep Multicolor Survey" (DMS, Osmer et al. 1998, and references therein), and EIS data for the South Galactic Pole (SGP, Prandoni et al. 1999). Then, the code, with the parameters fixed, was applied to the EIS data in the Chandra Deep Field South (CDFS, Arnouts et al. 2001; Vandame et al. 2001). 


\subsection{Paper I calibration}

The IMF, SFR, and AMR for the disc and halo were those already specified in Sects. 3.3 and 3.4 as being the default ones.

The disc component was described by a double-exponential in scale height and Galactocentric distance. The model did not have separate components representing the thin or thick disc. Instead the scale height for disc stars was a function of age, and was parametrized as in Eq. (7). The parameter values in this equation were not the same as in Rana \& Basu (1992) namely $z_{0}=95 \mathrm{pc}, t_{0}=0.5 \mathrm{Gyr}$ and $\alpha=(2 / 3)$, since this does not fit very well the derived scale height of "thick", "old", "intermediate" and "young" disc components as derived by Ng et al. (1997). Their results are described by $z_{0}=95 \mathrm{pc}, t_{0}=$ 4.4 Gyr and $\alpha=1.66$, which was adopted in Paper I.

Since none of the six DMS fields, nor the CDFS and SGP, contains a bulge component, this population was not included.

The Sun was assumed to be $15 \mathrm{pc}$ above the Galactic Plane (Cohen 1995; Ng et al. 1997; Binney et al. 1997) and the distance of the Sun to the Galactic Centre was assumed to be $8.5 \mathrm{kpc}$.

With these input ingredients fixed, the halo oblateness (and local halo number density) was derived by fitting the number of halo stars, defined by (in the Johnson-Cousins system) $0<$ $B-V<0.7$ in the range $20<B<22$ and $0<V-I<0.8$ in the range $18<I<20$, in these seven fields and was found to be $q=0.65 \pm 0.05$. This value was smaller than the value of $0.8 \pm 0.05$ quoted by Reid \& Majewski (1993), but Robin et al. (2000) could not exclude a spheroid with a flattening as small as $q=0.6$ and Chen et al. (2001) derived $q=0.55 \pm 0.06$.

The disc radial scale length (and local disc number density) was derived by fitting the number of disc stars, defined by $1.3<$ $B-V<2.0$ in the range $20<B<22$ and $1.8<V-I<4.0$ in the range $18<I<20$, and was found to be $h_{\mathrm{R}}=2800 \pm$ $250 \mathrm{pc}$. This was in agreement with the lower limit of $2.5 \mathrm{kpc}$ (Bahcall \& Soneira 1984) and the work of Zheng et al. (2001) on M-dwarfs who derived $h_{\mathrm{R}}=2750 \pm 160 \mathrm{pc}$ and Ojha (2001) who derive $h_{\mathrm{R}}=2800 \pm 300 \mathrm{pc}$ for the thin disc.

The model with these parameters was then used to estimate the stellar counts in the CDFS field, yielding a fairly good fit of the UBVRIJK number counts, CMDs, and colour distributions. The model with these parameters was also used by Marigo et al. (2003) to successfully predict the foreground population towards the LMC in JHK.

\subsection{Distribution of distance moduli}

It is very instructive to look at the characteristic distributions of distance moduli, $\mu_{0}=5 \log r-5$, for this model calibration. Were all stars in a given field - even the dimmest ones - possible to be observed, such distribution would be proportional to the integral of the quantity $\rho r^{2}$ (see Eq. (1)) over small bins of distance modulus. We show these quantities as evaluated for the line of sight of the North Galactic Pole (NGP) in Fig. 5. In the case of the disc, we separate the profiles coming from different ages (i.e. different scale heights) spaced by 1 Gyr. As can be noticed, a simulation of the NGP - if not constrained by any limiting magnitude - would contain increasing numbers of disc

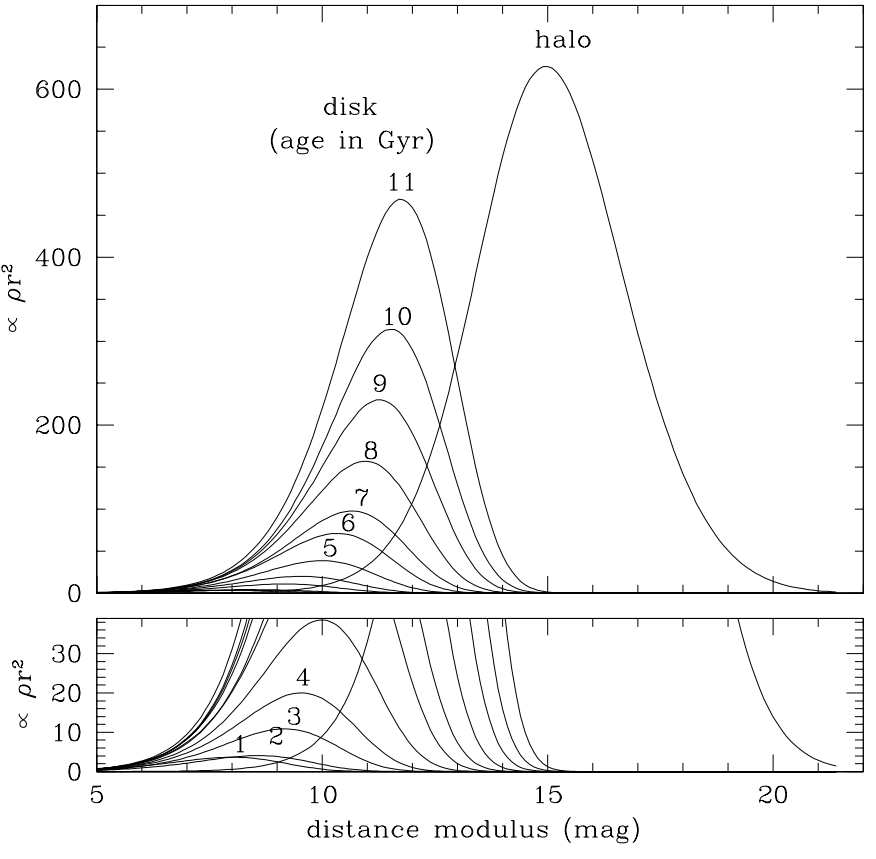

Fig. 5. Distribution of stellar distance moduli in the simulation corresponding to our initial calibration, in a conic bean towards the NGP. This is shown for 11 disc components of increasing age - at steps of $1 \mathrm{Gyr}$, as labelled - and for the halo. The top panel shows all the curves, whereas the bottom one expands the vertical axis in order to detail the profiles for ages younger than $5 \mathrm{Gyr}$. It can be noticed that younger disc components are found at lower mean distances (peaking from say $\mu_{0}=8$ to 12 as the age increase), whereas the halo stars are found with a nearly-Gaussian distribution of $\mu_{0}$ which peaks at about 15. The characteristic shapes of these distributions result from two competing trends in Eq. (1): the quadratic increase of the geometrical factor $r^{2}$, and the almost-exponential decrease of the density $\rho$ with distance to the Galactic Plane.

stars as we go to older ages, and at increasing mean distances (from $\mu_{0} \simeq 6$ to 12 as the age goes from very young to $11 \mathrm{Gyr}$ ). For each age considered, the disc distribution of $\mu_{0}$ looks like an asymmetric curve with a slow increase followed by a faster decay. The $\mu_{0}$ distribution of halo stars, instead, looks like a single Gaussian of mean $\mu_{0} \simeq 15$.

As a rule, we can conclude that halo stars dominate star counts at very large distance moduli $\left(\mu_{0} \gtrsim 13\right)$, whereas intermediate-age to old disc stars would dominate counts at most "intermediate distances" ( $\mu_{0}$ from say 9 to 13 ). Only at very short distance moduli $-\mu_{0} \lesssim 9$, i.e. in the immediate Solar Neighbourhood - can the young disc stars make a sizeable contribution to the star counts. Alternatively, one has to look at lower galactic latitudes to see a higher contribution from the young disc.

Of course, the situation gets more complex as we consider the limiting magnitudes that are present in any survey, and that favour the detection of the few closest stars in spite of the many distant ones. Anyway, the present Fig. 5 shows the type of stars which make the major contribution depending on the depth of a given survey. This information is relevant for the discussion presented in the next section. 


\section{Recalibration and fine tuning}

Since Paper I, we have improved many aspects of TRILEGAL, and checked the model predictions with additional datasets. This has forced some changes in the model calibration, as will be detailed in the subsections below.

\subsection{Summary of recent changes}

First of all, we opted for a better temporal accuracy as given by $N_{\mathrm{d}}=100$ (see Sect. 3.6) instead of the $N_{\mathrm{d}}=10$ adopted in Paper I. $N_{\mathrm{d}}=100$ implies a virtually continuous change of thin disc geometry with age, which in itself represents a novelty in star count models.

Second, we have adopted a more realistic metallicity distribution for halo stars, namely the one measured by Ryan \& Norris (1991). The Sun's height above the disc was corrected to 24.2 pc (cf. Maíz-Apellániz 2001).

So far, these changes have just a minor impact in our final number counts. Major revisions instead resulted from our attempts to reproduce 2MASS and Hipparcos data, not considered in Paper I. Without entering in a detailed description of all attempts carried out to fit the models to the data, here we describe the main arguments used in establishing our final calibration. They are:

1) the Paper I calibration, although in excellent agreement with deep star counts and with the constraints from Hipparcos, presented systematically too few stars at intermediate magnitudes, of say $K \sim 14$, as compared to 2MASS data. The deficit was small for high latitudes, reaching factors of about 2 at $b \sim 10 \mathrm{deg}$. This calls for an decrease in the slope of the LFs;

2) star counts at faint magnitudes are determined essentially by the halo plus the oldest disc components (those with highest scale heights, see Fig. 5). A way to decrease the LF slope is to reduce the contribution of the oldest disc components, and then compensate the reduction in total number counts by increasing the total disc SFR. We perform such an operation in the following way: the age scale of the thin disc SFR is changed by a constant factor of 0.8 (or -0.1 dex in $\log t$ ), so that the oldest disc component is found at an age of $9 \mathrm{Gyr}$, and with scale height of $h_{z}=603 \mathrm{pc}$;

3) this change in the age (and height) scales of the disc is quite satisfactory in predicting star counts in CDFS, DMS, and 2MASS, but now fails by predicting twice as many stars as sampled by Hipparcos. This local sample is dominated by relatively younger stars, of ages $\lesssim 2.5 \mathrm{Gyr}$ (as indicated by Fig. 5) and $h_{z} \lesssim 150$ pc. In order to eliminate this discrepancy, we simply change the vertical distribution of thin disc stars, from the usual exponential to a squared hyperbolic secant law with twice its scale height, i.e.

$\exp \left(-|z| / h_{z}\right) \rightarrow 0.25 \operatorname{sech}^{2}\left(0.5 z / h_{z}\right)$.

In this way, star counts at deep and intermediate magnitudes do not change by much, while the local one is reduced to the level of the observed values.

The assumption of a sech ${ }^{2}$ function instead of an exponential one is far from being arbitrary. Exponential laws are commonly assumed in star count models mainly because this is inferred from the surface brightness profiles in the discs of edge-on galaxies. But this observation does not refer to the inner part of the discs, which are normally obscured by dust lanes and are the site of ongoing star-formation. From the kinematic point of view, theory predicts that the density profile of isothermal discs do follow a $\operatorname{sech}^{2}$ law - then approaching an exponential law at high $z$, in accordance with observations. Of course, the disc of our Galaxy is not isothermal - as demonstrated by the observed increase of scale heights with the stellar ages -, but this latter theoretical aspect makes the sech ${ }^{2}$ law to be a better approximation than a simple exponential law.

Finally, in order to improve upon our final results for 2MASS and Hipparcos, he have modified the SFR of the thin disc: we have assumed that between 1 and 4 Gyr the SFR has been 1.5 times larger than at other ages. This causes a moderate impact in the distribution of stars in the $M_{V}$ vs. $B-V$ diagram, that can only be explored by means of Hipparcos data. In fact, such a change in the disc SFR has a negligible impact on deep fields and just a minor impact on 2MASS counts.

The normalization constants we derived imply

- a local surface density of ever-formed disc stars of $\Sigma_{\mathrm{d}}(\odot)=$ $59 M_{\odot} \mathrm{pc}^{-2}$, a number that compares well with the present dynamical surface density of matter in the Solar Cylinder (56 $\pm 6 M_{\odot} \mathrm{pc}^{-2}$ cf. Holmberg \& Flynn 2004). We find reassuring that these two quantities, being very different in their nature, have the same order of magnitude. This would indicate an efficient conversion of baryonic matter into stars over the history of our disc;

- a local volume density of halo stars ever-formed $\Omega_{\mathrm{h}}(\odot)=$ $1.5 \times 10^{-4} M_{\odot} \mathrm{pc}^{-3}$.

Let us now present the results for this calibration, providing more details on the way the different data samples have been selected and modelled.

\subsection{Simulating deep fields: CDFS, DMS, and SGP}

A main characteristic of deep photometric surveys is the rich presence of galaxies, a significant fraction of which appears as point sources and cannot be easily distinguished from real stars. Thus, object classification by means of morphological and photometric criteria is of central importance in these fields. The reader is referred to Paper I and Hatziminaoglou et al. (2002) for a discussion of these aspects.

In this paper, we deal with 3 deep catalogues which, as far as possible, have been cleaned from contamination by galaxies.

\subsubsection{CDFS}

The first one is the CDFS stellar catalogue (Paper I), which points towards a relatively clean area centered in $(\ell=$ $220.0 \mathrm{deg}, b=-53.9 \mathrm{deg}$ ). According to Schlegel et al. (1998) maps, the reddening for background sources amounts to $E_{B-V}=$ 0.0148 or $A_{V}=0.0458$. We make use of $U B V R I$ data from the 5-passband catalogue covering $0.263 \mathrm{deg}^{2}$, and $J K$ from the 7passband catalogue covering $0.0927 \mathrm{deg}^{2}$. The data has been 

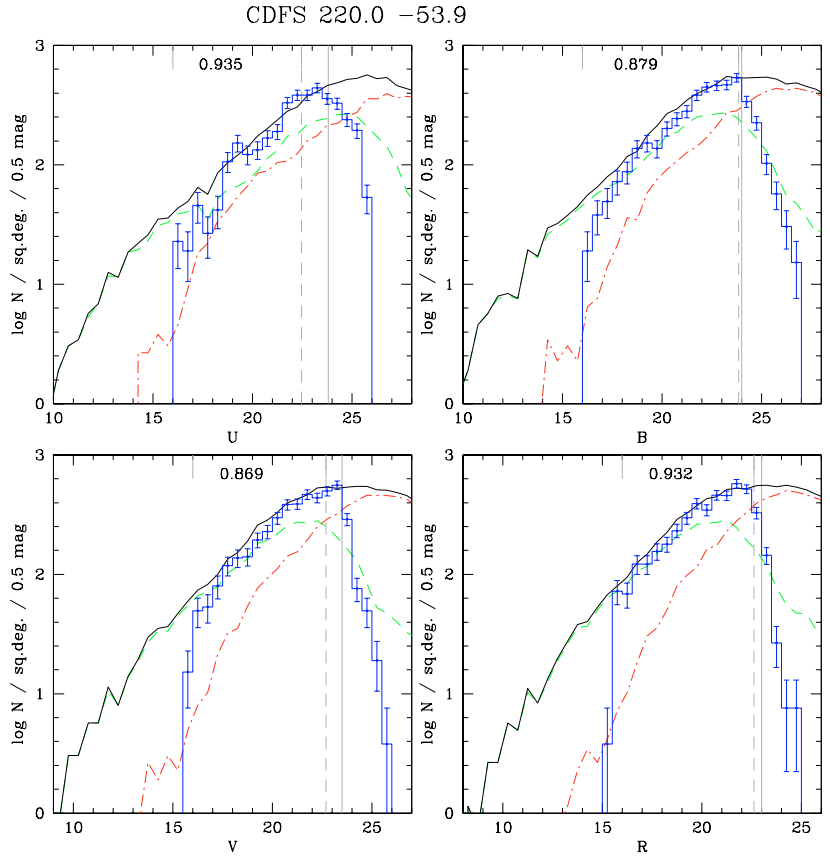
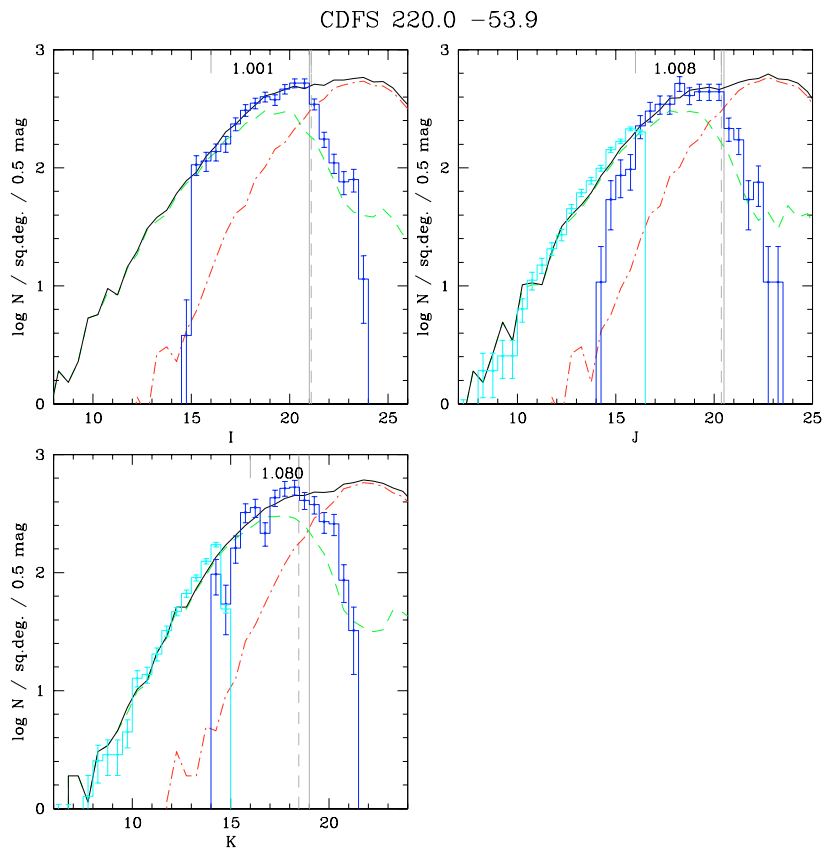

Fig. 6. Number counts as a function of magnitude. The histogram with error bars represents the data from the 7 and 5-passband CDFS stellar catalogues (Paper I) with poissonian errors. The dashed and continuous gray vertical lines indicate the magnitude limits for efficient morphological classification, MAG_STAR_LIM, and the 90 percent completeness limit, respectively. For the $J$ and $K$ plots, at brighter magnitudes $(J<16.5$ and $K<15$, respectively) we add the histogram corresponding to 2MASS $J K_{\mathrm{s}}$ data. The smooth lines refer to the model results for a region of much larger area and hence better statistics, computed with the same binning $(0.5 \mathrm{mag})$ as the data: they show separately the contribution of the halo (dot-dashed line), of the disc (dashed), and the total result (continuous line). At the top of the plot, we present the number ratio between observed and modelled sources as counted within the limits indicated by short gray vertical marks (the fainter limit coincides with MAG_STAR_LIM). Inside these magnitude limits, the comparison between the continuous line and histogram indicates the goodness of the model in reproducing the observed star counts.

cleaned from non-stellar objects according to the criteria and methods thoroughly discussed in Paper I. For this field, we simulate the galactic population for a $2.63 \mathrm{deg}^{2}$ region. Figure 6 shows the results in units of number of stars per unit $\mathrm{deg}^{2}$ and 0.5-mag intervals. As can be noticed, the agreement between modelled and observed counts is good, their ratio being comprised between 0.87 and 1.08 for all 7 pass-bands considered.

Particularly interesting is the comparison between simulated and observed number counts for stars in the interval $-0.4 \leq U-B \leq 1.2$. This subsample, according to the models, is completely dominated by disc stars at brighter magnitudes ( $V \lessgtr 18$ ), and then by halo stars at fainter magnitudes $(V \gtrsim 20)$; this is illustrated by the comparisons between observed and simulated CMDs shown in Paper I (see in particular the online version of their Fig. 5). Hence, by fitting number counts in this particular subsample we can be sure to be correctly modelling the relative proportion between halo and disc densities. Moreover, a "blue subsample" defined in this way is composed mostly by stars in well-modelled evolutionary stages - i.e. main sequence stars of moderately high $T_{\text {eff }}$ - and excludes the hot white dwarfs and the reddest very-low mass stars, for which the reliability of present-day evolutionary and spectral models could still be questioned.

Such a comparison is presented in Fig. 7. As can be noticed, the blue subsample presents good evidence that the ratio between halo and disc densities is well represented for this lineof-sight. The most significant discrepancy that appears at this point is a moderate excess of halo stars at $B \simeq 23$. As demonstrated in Paper I, this is a magnitude interval in which number counts become sensitive to the very low-mass IMF. In fact, we have verified that a better agreement with observations turns out if we artificially eliminate halo stars with $M_{\mathrm{i}} \lesssim 0.2 M_{\odot}$ from our models. However, we prefer not to draw any strong conclusion from this test, since $B \simeq 23$ is also close to both the limit for efficient morphological classification, MAG_STAR_LIM, and the 90-percent completeness limit of CDFS data.

\subsubsection{DMS}

The Deep Multicolor Survey by Hall et al. (1996) and Osmer et al. (1998) provides deep UBVR'I75 and I86 data for 6 different fields of $|b| \gtrsim 30 \mathrm{deg}$. From their catalogue, we eliminated the objects that are likely not to correspond to stars, i.e. those marked as "galaxy", "noise", "diffuse object", or "long object" in any of the DMS passbands.

Figure 8 shows the $U B V R$ results as compared to our models. As shown in the figure, if we limit the analysis to magnitudes between the DMS "upper limiting" and the "threshold" one - an interval almost free from saturation, incompleteness and contamination effects - there is a good overall agreement in the number counts between the data and our model. With the exception of DMS field 21, the largest discrepancies reach about 10 percent in number counts. 

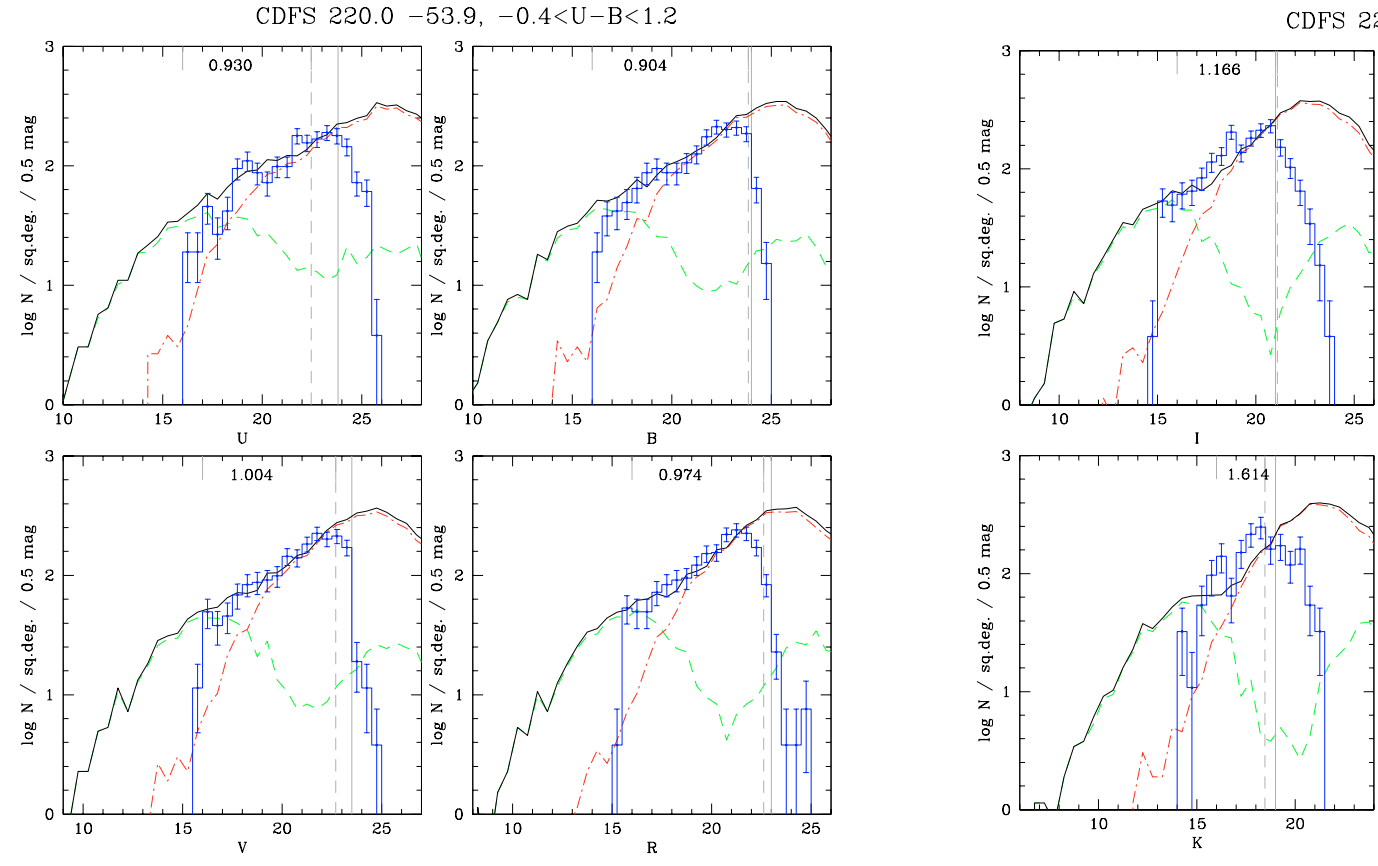

CDFS $220.0-53.9,-0.4<\mathrm{U}-\mathrm{B}<1.2$
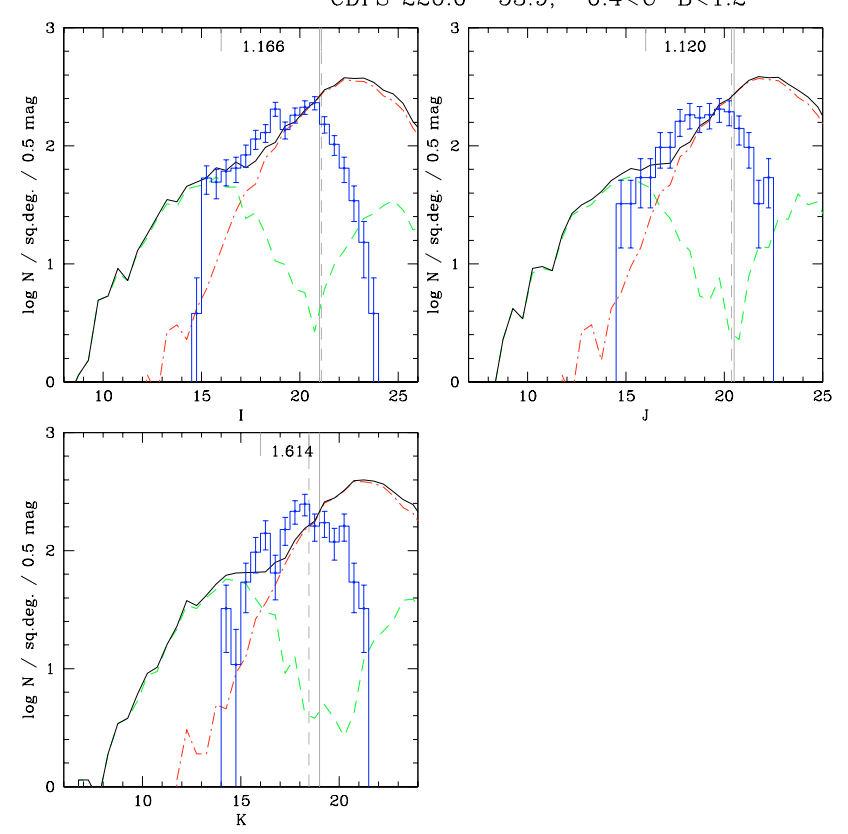

Fig. 7. The same as Fig. 6, but now limiting data and models to the "blue subsample" with $-0.4 \leq U-B \leq 1.2$, which is dominated by the disc at brighter magnitudes and by the halo at the faintest. To help in the comparison, the scales are kept the same as in Fig. 6 . The comparison between data (histogram with error bars) and models (continuous line), inside the magnitude interval delimited by the vertical gray marks, probes whether the models correctly predict the number ratio between halo and disc stars (shown separately by dot-dashed and dashed lines, respectively).

Again, the comparison between simulated and observed number counts for stars in a "blue subsample" - now defined in the interval $-0.4 \leq U-B \leq 0.8^{4}$ - are important for verifying whether we have the right proportions between disc and halo stars. A careful examination of Fig. 9 reveals that this is indeed the case, but for DMS field 21, where, apparently, simulations contain too few disc stars. Field 21 is the innermost of DMS fields, pointing to a Galactic region for which actually our model calibration indeed presents the largest problems (see Sect. 5.3).

\subsubsection{SGP}

The South Galactic Pole (SGP) as observed by EIS (Prandoni et al. 1999; Zaggia et al. 1999) presents $B V I$ data for an effective area of $1.21 \mathrm{deg}^{2}$ centered at $\ell=306.7 \mathrm{deg}, b=-87.9 \mathrm{deg}$. Figure 10 shows the comparison between the data and our models. This time, the shape of the observed counts are, in general, fairly well reproduced, except that the amplitude of our model is twice as large as observed. We did not find any obvious way of reducing this discrepancy, without spoiling the excellent agreement we find for the other fields considered in this paper - including 2MASS data for the SGP itself. Also, the lack of $U$-band data prevent us from analysing a blue subsample essential in order to investigate the source of the discrepancies.

\footnotetext{
${ }^{4}$ The Johnson $U-B$ colour of DMS separates less the red and blue sequences typical of field CMDs, than the $U-B$ colour of CDFS - this latter being based on ESO WFI filters. This is the reason why we have used a smaller interval of $U-B$ to define the blue subsample of DMS data, than for CDFS.
}

\subsection{Simulating $2 M A S S$ data}

As seen above and also in Paper I, the deep simulations of DMS, SGP and CDFS data are ideal for probing the relative proportions between halo and disc components, as well as the shape of the halo and the IMF of the disc. For better probing the disc and its details (spiral arms, dust lanes, warps, etc.), shallower surveys covering larger areas are better suited. However, if one wants to avoid complications caused by dust, we should consider only counts at the infrared. In this context, 2MASS constitutes an invaluable dataset: it covers the all sky in $J H K_{\mathrm{s}}$ for magnitudes as faint as $J \sim 17$.

From the 2MASS All-Sky Data Release Point Source Catalog we have selected the sub-sample obeying the so-called "2MASS level 1 science requirements" (see the User's Guide in Cutri et al. 2003). In practice, these criteria refers to stellar sources falling outside of tile overlap regions, and of high photometric quality (namely $S / N>10$ and $\sigma<0.11 \mathrm{mag}$, bandby-band). For most of the sky - excluding the most crowded low-latitude and bulge fields - this subsample of 2MASS is essentially complete for magnitudes brighter than about 15 .

In the panels of Fig. 11, we show the complete results for two 2MASS fields, one of high latitude (the NGP at $\ell=$ $0 \mathrm{deg}, b=90 \mathrm{deg})$ and one of low ( $\ell=180 \mathrm{deg}, b=10 \mathrm{deg})$. Plots for symmetric fields - namely the SGP and the $(\ell=$ $180 \mathrm{deg}, b=-10 \mathrm{deg}$ ) ones - look very much the same and present similar number counts. The counts in these particular fields are very well reproduced by the model, over a wide range of magnitudes in all the 3 pass-bands of 2MASS. The reader can also notice that the $J, H$ and $K_{\mathrm{s}}$ diagrams contain essentially the same information, so that examining all of them may 

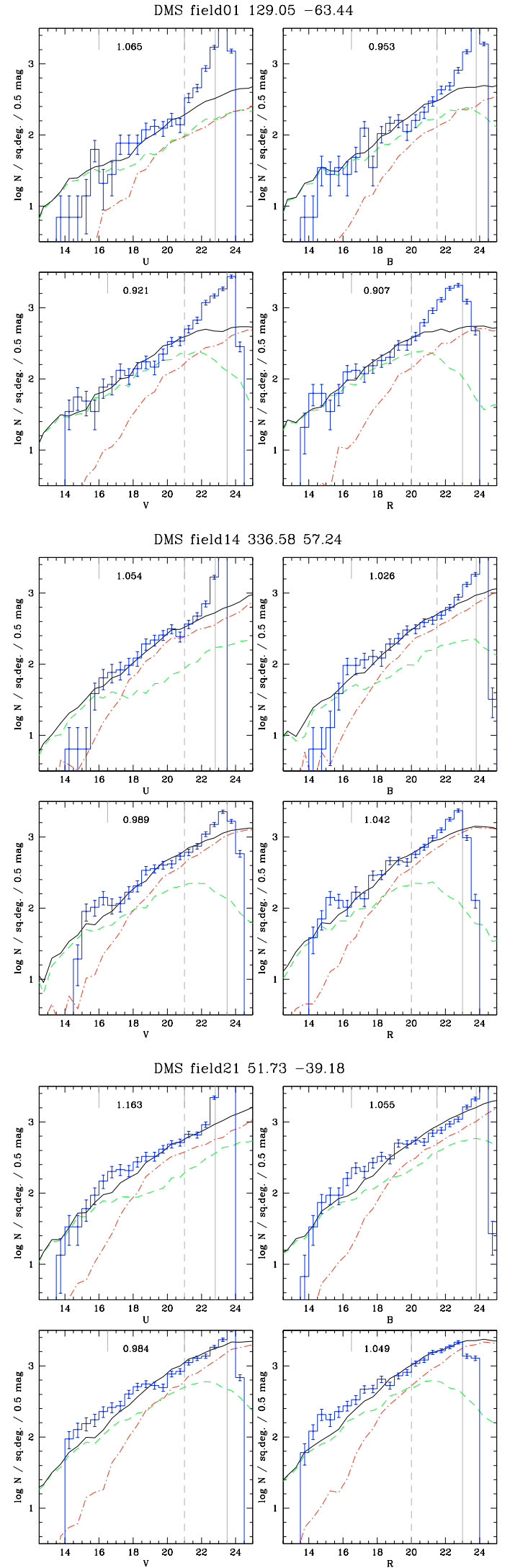

Fig. 8. The same as Fig. 6, but for the several fields of the DMS (Osmer et al. 1998), whose $(\ell, b)$ are given at the top of each panel. The data (histogram with error bars) excludes the objects that are likely not to correspond to stars. The gray vertical lines this time indicate the DMS "threshold magnitude" (dashed line) and the " $5 \sigma$ limiting magnitude" (also the 90 percent completeness magnitude; continuous line) as determined by Hall et al. (1996). The star counts ratio between model and data (shown in the upper part of each panel) is computed between the "upper limiting magnitude" (16.0 or 16.5, depending on the passband) and the threshold one. 

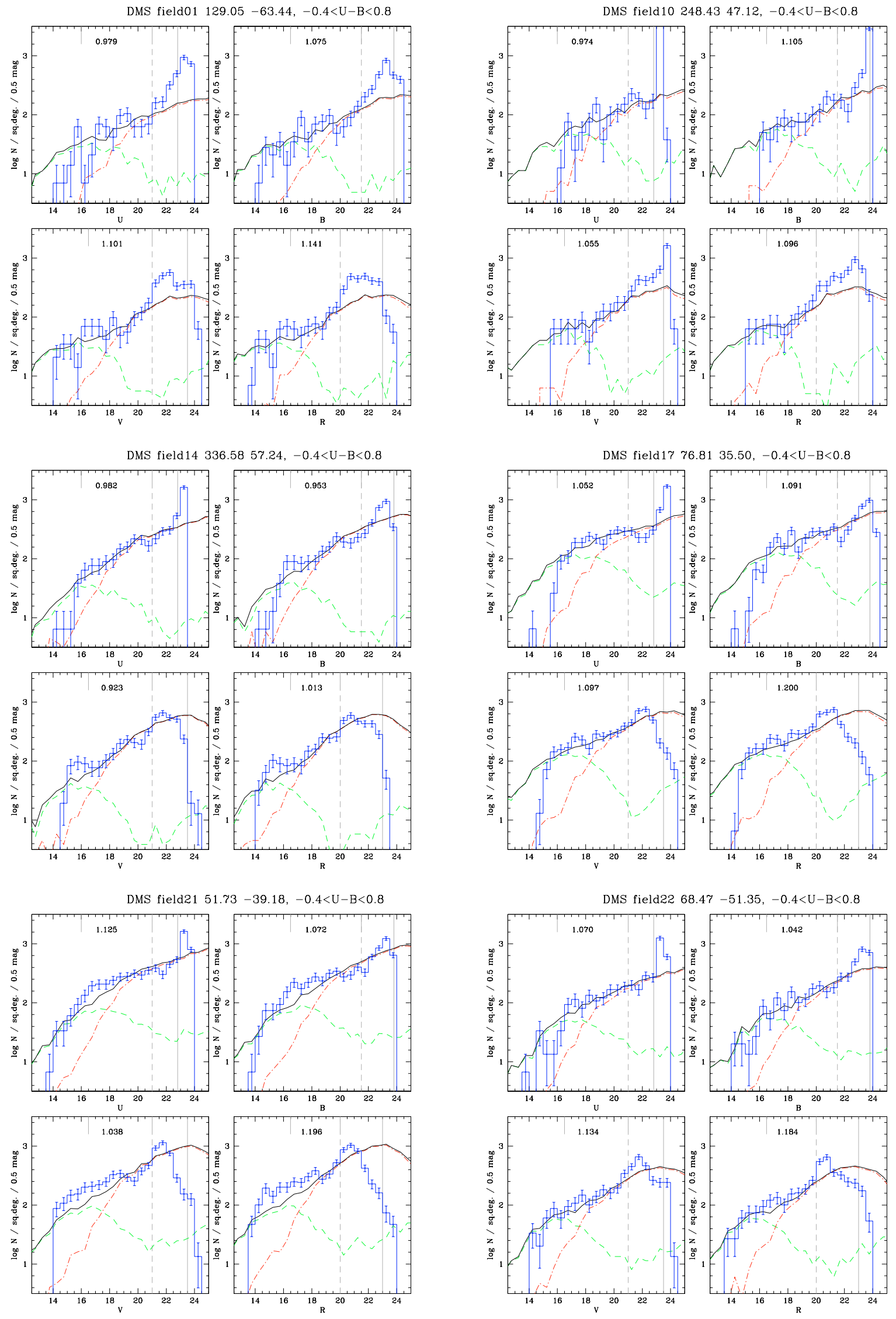

Fig. 9. The same as Fig. 8, but now limiting data and models to the "blue subsample" with $-0.4 \leq U-B \leq 0.8$. 

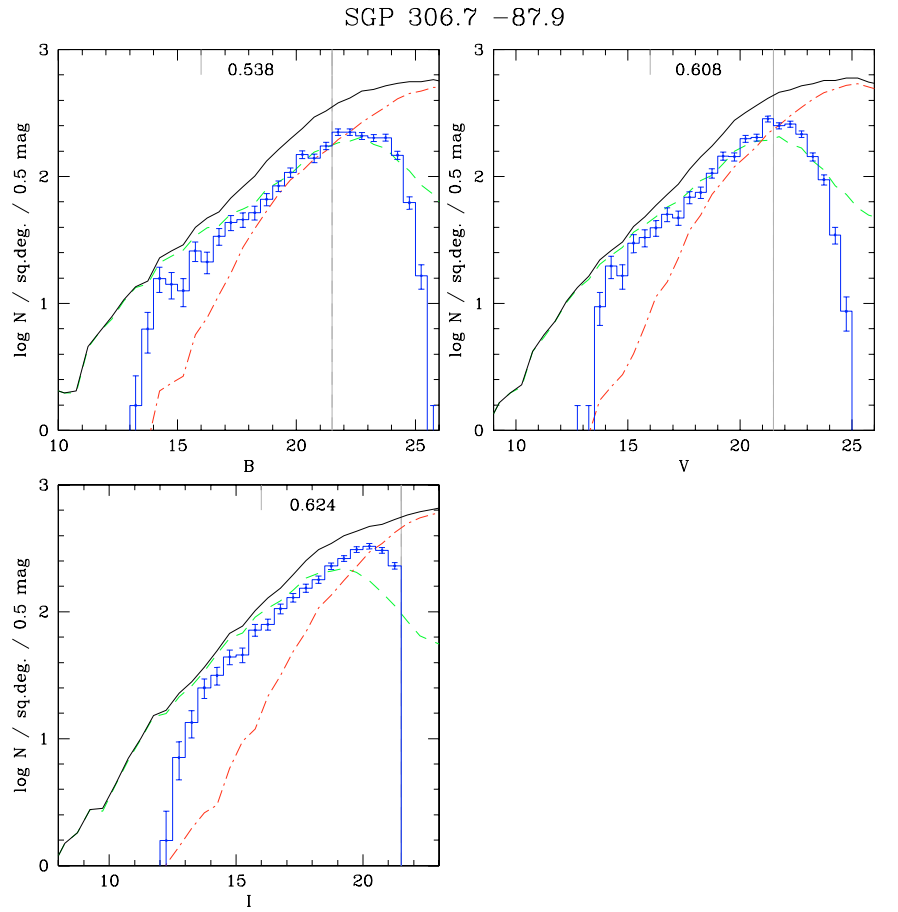

Fig. 10. The same as Fig. 6, but for the EIS-deep SGP data (Prandoni et al. 1999). The limits of reliability of the data were located, somewhat arbitrarily this time, at magnitudes 16 and 21.5 for all filters.

be redundant. For this reason and for the sake of conciseness, in the next figure we prefer to present just results for the $H$ band.

Figure 12 presents the $H$-band results for a series of 2MASS fields disposed along a great circle in the sky - the one at $\ell=0,180 \mathrm{deg}$, encompassing the Galactic centre, anticentre and polar regions. We show only the northern Galaxy fields, since results for southern fields are essentially the same.

The results are certainly encouraging. We are able to predict the correct number counts, with errors smaller than $\sim 30$ percent, for all fields located at least 10 degrees above the Galactic Plane, except for inner Galactic regions where the lack of a Bulge component - presently not included in the model becomes noticeable. Moreover, it is not to be excluded that the present description of the Galactic halo fails for small galactocentric distances, hence contributing to the discrepancies we find at inner galactic regions.

Remarkably, we find that for all 2MASS fields away from the Bulge we have analysed, disc stars make the bulk of the number counts. The halo contribution is almost negligible. However, we recall that halo stars contribute to make a particular feature in wide-area $J$ vs. $J-K_{\mathrm{s}}$ CMDs, namely the central of three vertical fingers discussed by Marigo et al. (2003), that are clearly present in 2MASS CMDs towards the LMC. This indicates that the halo component has its importance in analyses of 2MASS photometry.

In fields too close to the Galactic Plane, instead, our model predictions fail, as demonstrated by the first panel of Fig. 12 regarding the direction of the Galactic anticentre. One of the reasons for this failure is surely the too simplistic modelling of the dust distribution along the Galactic Plane. Improving this aspect of the model, however, is beyond the scope of the present paper.

\subsection{Simulating Hipparcos data}

Examining the stellar counts in the immediate Solar Neighbourhood is an obvious test for any Galaxy model. In fact, present models usually check whether their results are consistent with the observed "local stellar density", or with some similar parameter derived from Hipparcos data (see e.g. Robin et al. 2003). Of course, by using just a single density parameter as a constraint, we ignore the wealth of photometric information that is present in the data for nearby stars, that could tell us much about the distribution of stellar parameters in the disc. In order to start exploring this information, in the following we try to predict the counts of a local sample, using the Hipparcos dataset.

First of all, however, we should remind that our model in Paper I has been effectively calibrated on deep data $(V \gtrsim 15)$. For such deep surveys the effective distributions of distance moduli should approach the ones shown in Fig. 5, i.e. the samples are dominated by relatively far objects, at distance moduli ranging from $\mu_{0} \sim 7$ to 14 for the disc populations, and from $\mu_{0} \sim 12$ to 18 for the halo. It is then obvious that our previous model calibration has almost nothing to do with the very local sample of stars, i.e. the one observed at distances lower or comparable to $100 \mathrm{pc}$, and at bright magnitudes such as $V \lesssim 8$, that we will define below. Deep and local samples could even be considered, in terms of their stellar populations, as completely independent data samples.

The Hipparcos and Tycho catalogues (ESA 1997) have provided parallaxes with $\sim 10$ milliarcsec (mas) accuracy for several thousands of stars, together with accurate $B V$ photometry. The Hipparcos input catalogue was constructed in such a way that there are no clearcut criteria for defining volumelimited samples out of its data. This problem has been recognized by a number of previous authors. Hernandez et al. (2000) and Bertelli \& Nasi (2001), for instance, find it to be extremely difficult to define volume-limited samples containing enough stars for studying the SFR in the solar vicinity up to the oldest possible ages (see also Schröder \& Pagel 2003).

However, our aim in this paper is different from previous works. We consider a subsample of the Hipparcos catalogue to be good provided it is complete and could be simulated. Differently from the above-mentioned papers, we do not need to limit our simulations to stars being all contained in the same volume. Our sample can be selected by using a few simple criteria, based on the following realizations:

- the Hipparcos catalogue contains all stars brighter than $V_{\lim } \simeq 7$

- from them, all parallaxes $\pi$ higher than $\pi_{\text {lim }} \simeq 10$ mas have been measured;

- due to the presence of parallax errors, the sample defined by $\pi_{\text {lim }}$ comprehends a volume of radius $r_{\text {lim }}$ somewhat larger than $1 / \pi_{\text {lim }} \simeq 100 \mathrm{pc}$;

- among these stars, the bulk of binaries has been identified. 

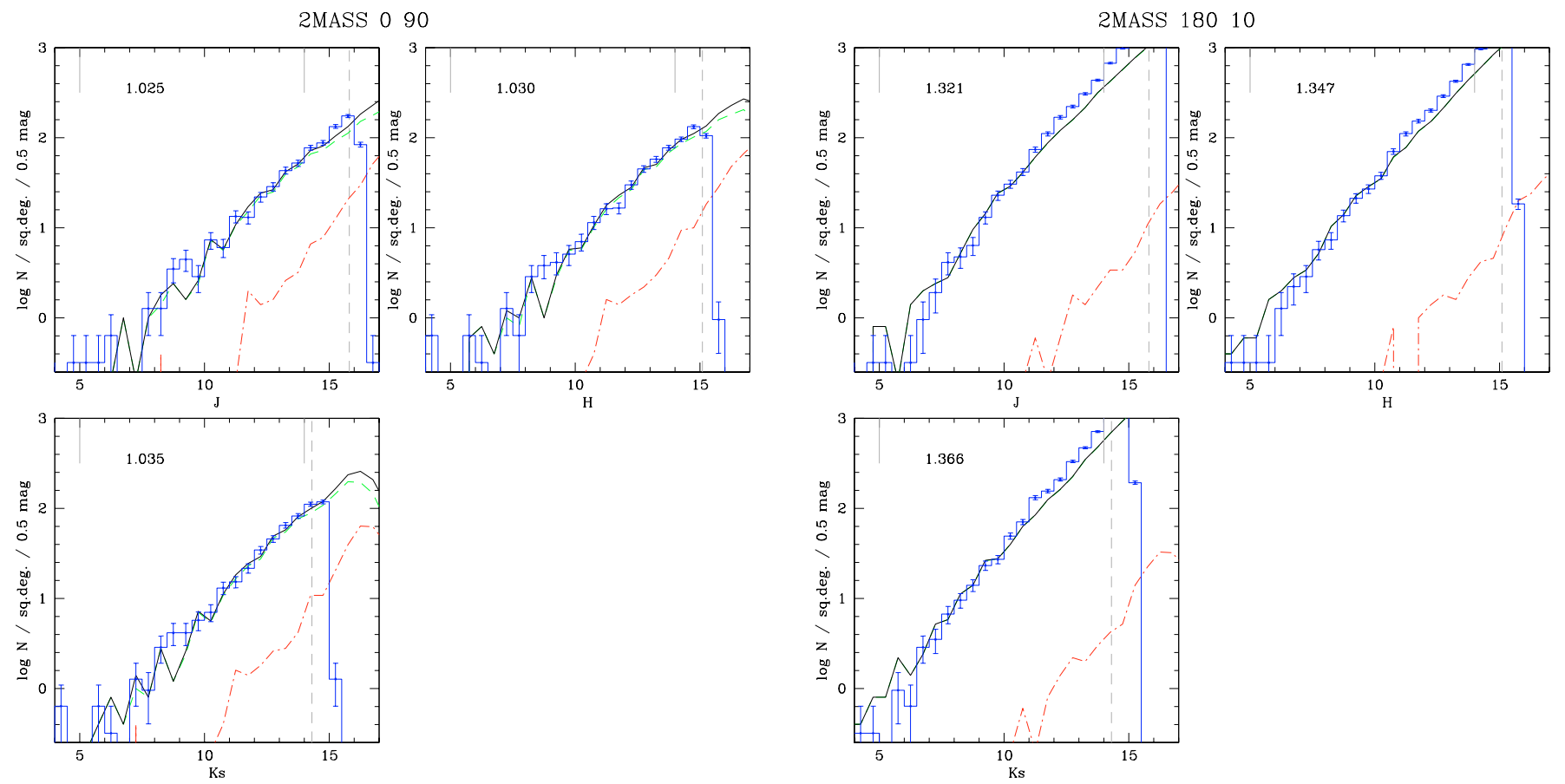

Fig. 11. The same as Fig. 6 , but for two sample fields of 2 MASS data: $(\ell=0 \mathrm{deg}, b=+90 \mathrm{deg})$ (the NGP), and $(\ell=180 \mathrm{deg}, b=+10 \mathrm{deg})$. Lines have the same meaning as in previous figures. In both cases, the disc component is responsible for the bulk of the number counts in the model.

Therefore, we select from the Hipparcos catalogue all stars with $V<V_{\lim }$ and $\pi>\pi_{\text {lim }}$, and not classified as binaries of any kind. $V_{\lim }$ and $\pi_{\lim }$ are kept as parameters. Initially, we have conservatively adopted $V_{\lim }=7$ and $\pi_{\lim }=10$ mas, which corresponds to $r_{\mathrm{lim}}>100 \mathrm{pc}$.

We then simulated this local sample using the TRILEGAL code. To do so, we have generated synthetic samples up to a distance $r_{\mathrm{lim}}=200 \mathrm{pc}$. This is large enough to include all stars that, due to parallax errors, will later be scattered to apparent distances closer than $100 \mathrm{pc}$.

The simulated true physical distances $r_{0}$ are first converted in the true parallax $\pi_{0}=1 / r_{0}$. The simulated parallax errors $\delta \pi$ (described in the Appendix) are then added to $\pi_{0}$ so as to generate the "observed" parallaxes and distances, $\pi$ and $r=1 / \pi$. The "observed" absolute magnitude is then derived by the usual formula, $M_{V}=V-5 \log r+5$. Extinction has been ignored, since its effect inside a radius of $200 \mathrm{pc}$ is negligible.

The results of this exercise can be seen in Fig. 13, for the conservative choice of $V_{\lim }=7$ and $\pi_{\lim }=10$ mas. In the lefthand panel, we compare the simulated and observed $M_{V}$ vs. $B-V$ diagram. The agreement between simulated and observed samples is striking. It can be noticed that models describe very well both the location and width of the main sequence all along from $M_{V} \sim 9$ to -4 . Particularly good is the description of the left boundary of the MS, very well marked because it is produced by stars in their phase of slowest evolution, when they depart from their ZAMS to the right in the HR diagram. Regarding the MS width, we know from stellar models that it is affected essentially by two factors: the assumed metallicity dispersion (or equivalently the AMR in our models) and the efficiency of convective core overshooting for $M>1 M_{\odot}$ stars.
The good agreement between models and simulations seems to indicate that both these ingredients are well described in our models. Of course, before considering the implications that this result may have for the disc AMR and for the theory of stellar evolution more quantitative comparisons would have to be made. However, such a discussion is beyond the scope of the present paper.

Regarding the evolved stars (subgiants and red giants), the agreement is also very good: we can notice the right width of the subgiant and lowest part of RGB; the clumping of coreHe burning stars in the right location; the bifurcation of the red giants above the clump into two loose broad sequences: a vertical one made of intermediate-mass core-He burners and the inclined one, going to the red, made of genuine first-ascent RGB and early-AGB stars.

As can be seen, the simulation predicts about the same star counts as observed: the total number of objects in both panels is 4085 and 4182 for the data and models, respectively.

The right panels show the corresponding distributions of apparent distance $r$ and derived absolute magnitude $M_{V}$ (continuous lines). The dashed lines refer to the subsample of subgiants and giants, defined by the stars with $M_{V}>6.82 \times$ $(B-V)-2$.

There, although a first look indicates a good overall level of agreement between models and data, some discrepancies become apparent. In the histogram of $r$, the most noticeable one seems to be a modest excess of simulated stars at $r \sim 80 \mathrm{pc}$, that amounts to about 20 percent. Since the volume sampled in the simulation is very small, we consider that such a discrepancy is unlikely to be derived from inhomogeneities in the local distribution of stars; more likely, a better simulation of 

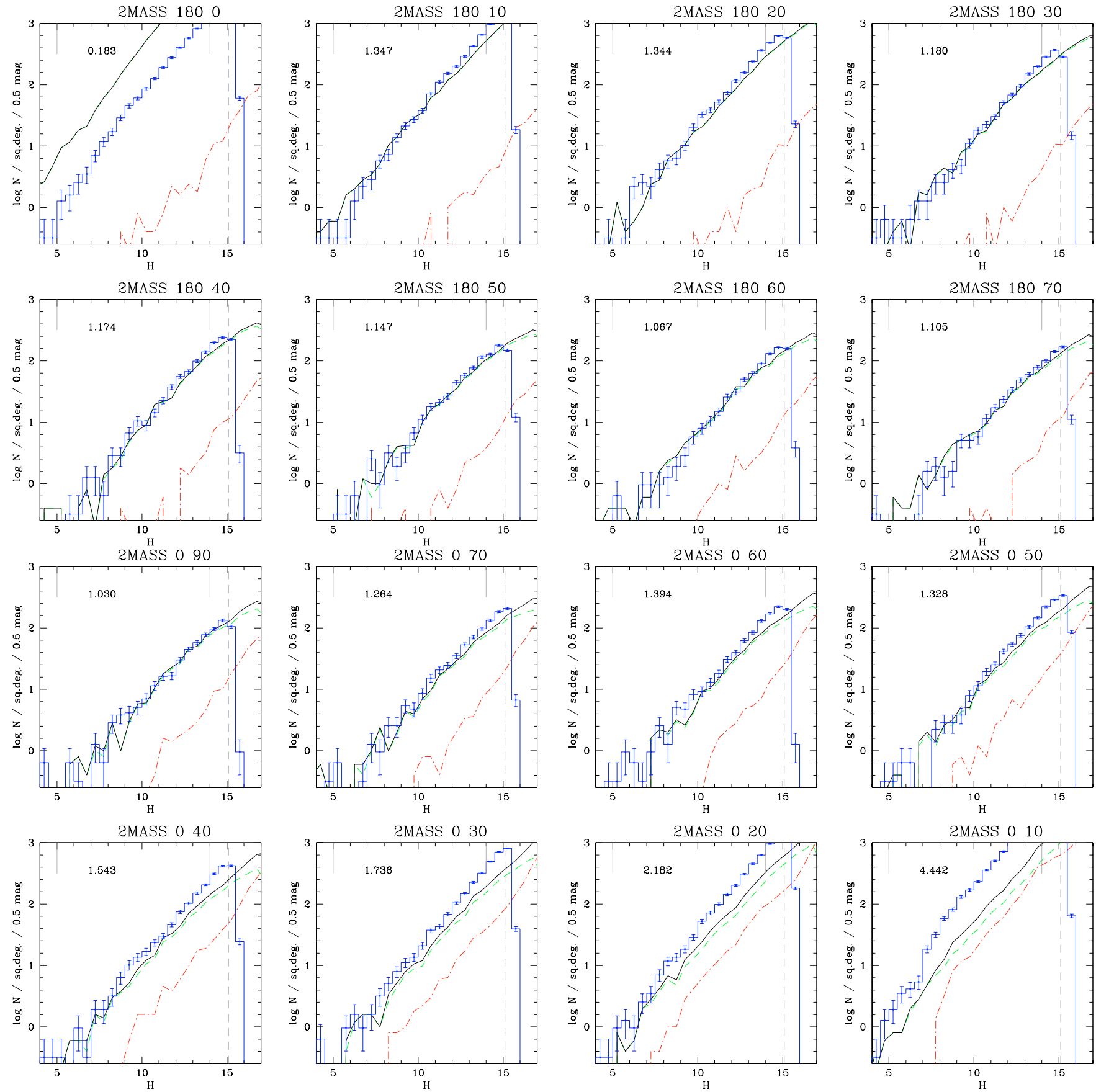

Fig. 12. The same as Fig. 11, but limited to the $H$-band and for a series of fields disposed along the $\ell=0,180$ deg great circle in the sky. Just the northern part of the circle is presented, since the results are similar for the southern fields. For the fields pointing towards the outskirts of the galactic bulge, $(\ell=0, b=+20)$ and $(\ell=0, b=+10)$, the bulge population is clearly seen as an increase in stellar counts at $H \geq 8$, caused by the bulge RGB, which is not accounted for by our disc+halo model.

Hipparcos parallax errors could improve the models in this particular range of distances (parallaxes). There is also a modest deficit of simulated stars in the smallest distances (from 15 to $35 \mathrm{pc}$ ), amounting again to about 20 percent, which however does not appear among the subgiants and giants. This again might indicate a problem in the simulation of parallax errors for the faintest stars. On the other hand, the spike of observed star counts at $r=45 \mathrm{pc}$ is to be assigned to the Hyades cluster, which was not considered in our simulation.
The histogram of number counts against $M_{V}$ indicates, once again, some modest discrepancies, that are however statistically very significant. The most important one consists on an excess of simulated bright stars accompanied by a deficit of the faintest ones. We have performed a KS-test comparison between the two $M_{V}$ distributions, and find that the probability of them being drawed from the same distribution would be highly increased - from its present close-to-zero value up to about 0.3 - if our models were shifted by $0.26 \mathrm{mag}$ in $M_{V}$. We think 

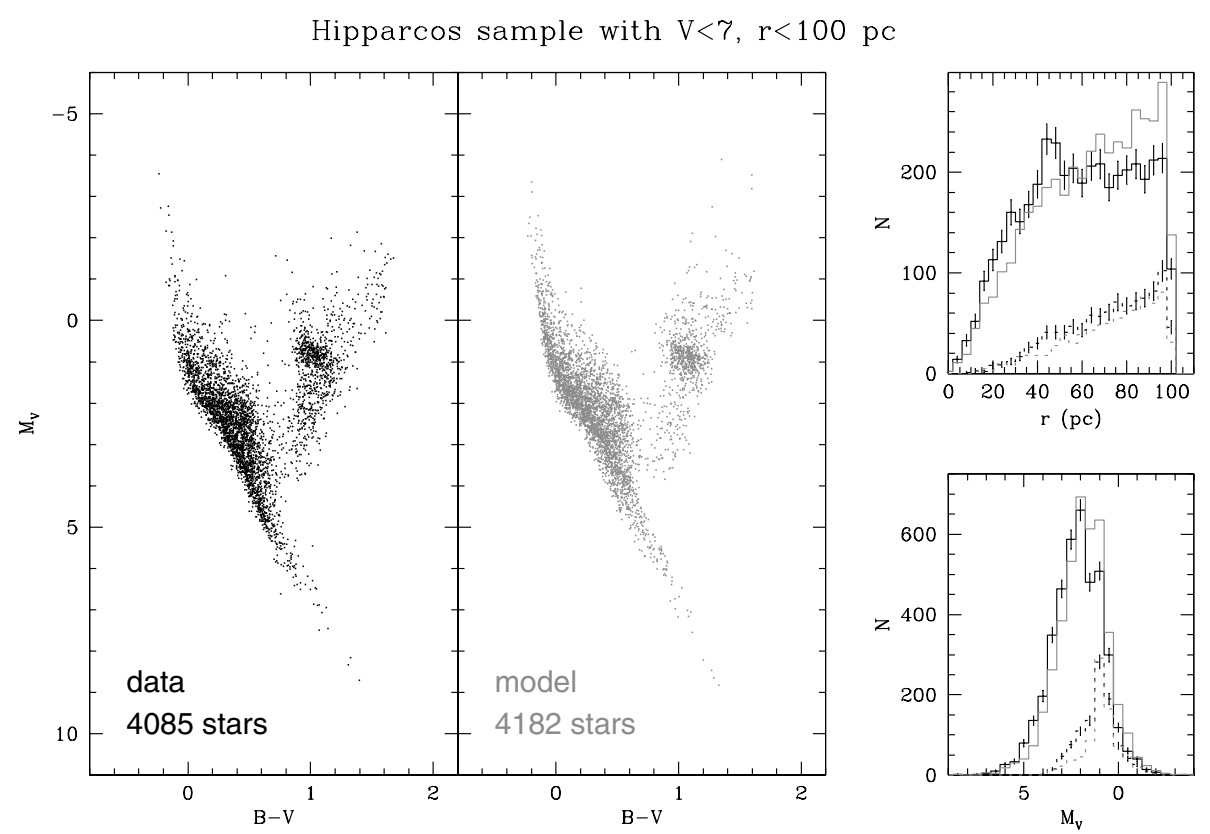

Fig. 13. The several panels show our Hipparcos simulation (grey) versus the real data (dark). On the left panels, we have the $M_{V}$ vs. $B-V$ diagram of both samples (observed and simulated) limited to a parallax of $\pi<10$ mas (apparent distance $r=1 / \pi<100 \mathrm{pc}$ ) and an apparent magnitude of $V<7$. The agreement between simulated and observed samples is remarkable for most regions of the diagram. The lines in the right panels show the corresponding distributions of apparent distance $r$ (top) and derived absolute magnitude (bottom), for both the total samples (continuous lines) and the subsample of subgiants and giants (dotted lines). For the data histograms, error bars denote the standard error $(\sqrt{N})$ of a Poisson distribution, and serve as a guide to the comparison between model and data. As can be noticed, the simulation predicts about the same total star counts as observed, with just a 2.5 percent excess of model stars. Looking at the right panels, however, we notice a significant excess of simulated stars at $r \simeq 80 \mathrm{pc}$, a deficiency at $r \simeq 20 \mathrm{pc}$, and the obvious failure to reproduce the spike at $r=45 \mathrm{pc}$ that corresponds to the Hyades cluster. Moreover, in the comparison of number counts as a function of $M_{V}$, there seems to be an excess of bright stars and a deficit of faint ones.

however that applying such a shift whould not be justified, and it could not be easily translated onto a physical interpretation (i.e. shifts in the $M_{V}$ distribution can be forced by using models with a corrected photometric zero-point, different IMF, different SFR, modified prescription for simulating parallax errors, or a combination of all these effects).

To conclude, we remark that all the above-mentioned discrepancies are, from the statistical point of view, highly significant, since they refer to samples containing large numbers of stars. They may be indicating points where the present models can be improved. They however do not invalidate the present model calibration, for a series of reasons: first of all, many of the discrepancies are suspected to result from the imperfect simulation of parallax errors; second, when one deals with normal star counts data, fine details (i.e. those seen at $\sim 0.25 \mathrm{mag}$ resolution) of the $M_{V}$ distribution become of little relevance since stars are dispersed over a large range of distance moduli; third, the most relevant comparison regards the total star counts that are directly linked to the local density of stars: in our case they are very well predicted, to within 5 percent. Reaching such a result for the star counts is already remarkable, whereas reaching a statistically-robust comparison with Hipparcos, in all its details, may be still far from reach. In this regard, the present work represents just the first attemp.

Figure 14 presents the same as Fig. 13, but for a slightly deeper sample, of $V_{\lim }=8$ and $\pi_{\lim }=10$ mas. The effect of selecting a deeper $V_{\lim }$ is that more stars with $M_{V}>2$ are included in the sample, then increasing the contribution of intermediate-age to old stars (in their main sequence, subgiant branch, and initial RGB evolution) to the number counts. In this case, the star counts are 8055 and 7640 for data and models, respectively. The discrepancies between model and observations seem slightly increased, as expected since we are including data for which the completeness starts to become an issue, and for which parallax errors are slightly larger with respect to the previous $V<7$ sample.

\section{Discussion and conclusions}

We have presented a new code for simulating the photometry of Galaxy fields.

The code has been calibrated by predicting counts in a variety of stellar surveys, that comprehend some very deep multipassband catalogues cleaned from galaxies (CDFS, DMS, SGP), the "intermediate-depth" near-IR point source catalogue of 2MASS, and the very local stellar sample derived from Hipparcos catalogue.

The results are certainly satisfactory, since we have demonstrated that the predicted star counts agree well with the observed ones. The typical discrepancies are smaller than 30 percent for most of the sky, and inside the estimated magnitude limits of reliability of the observed star counts. This agreement 

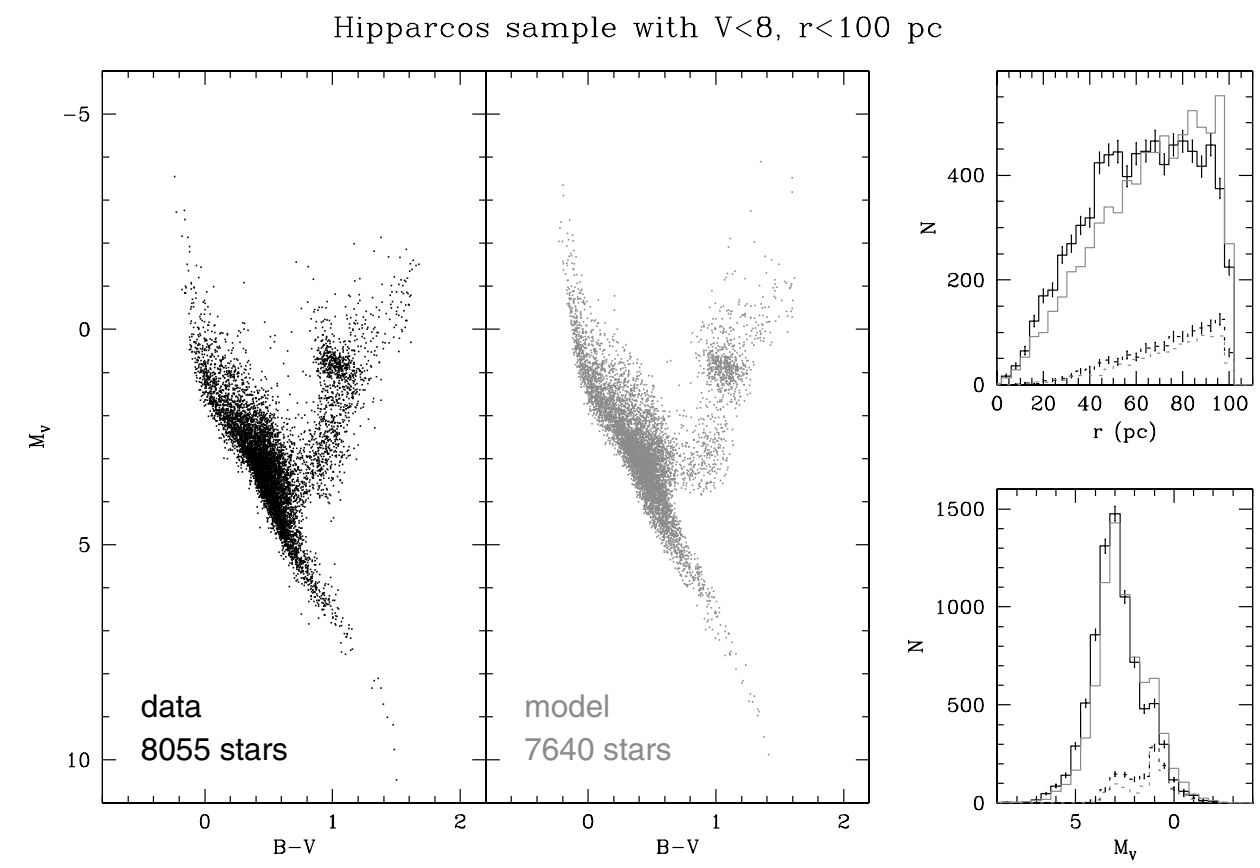

Fig. 14. The same as Fig. 13, but for samples limited to an apparent magnitude of $V_{\lim }<8$. Notice the increased number counts of stars with $M_{V}>2$.

is remarkable, when we consider the wide ranges of magnitudes, passbands (from $U$ to $K$ ) and sky positions that were considered in this work.

The major discrepancies were found for: (1) inner Galactic fields, located within about $30 \mathrm{deg}$ from the Galactic Center, for which we largely underestimate the number counts. Part of this discrepancy can be attributed to the lack of a bulge component in the present model, but probably a better modelling of the inner disc and halo densities is also necessary. (2) Lowlatitude fields, with $|b| \lesssim 10 \mathrm{deg}$, which probably require a detailed modelling of the distribution of dust and recent star formation along the disc. Finally, (3) the SGP as observed by EIS, for which we predict twice as many counts in the optical as observed. In this case, the origin of the discrepancy could not be identified.

Note that the present model calibration is not yet fully optimized and is likely not to be unique, in the sense that other choices for the stellar densities and star formation histories of Galaxy components might produce similarly good results. In fact, the question arises whether TRILEGAL could be adapted to find, in an objective way, a maximum-likelihood solution for the functions $\rho, \psi, \phi$, etc. - using a database of few highquality multiband catalogues covering several regions of the sky and with a large range in depth. The answer is likely yes, but the way is certainly not straightforward. Such a work would imply at least the following steps: (a) finding a zerothorder calibration producing "acceptable" results, which is actually the step performed in this paper; (b) establishing a robust likelihood criterium for comparing the resulting models with the stellar data; (c) establishing a numerical algorithm to migrate from the zeroth-order to improved maximum-likelihood solutions (see for instance $\mathrm{Ng}$ et al. 2002); (d) exploring the problem of uniqueness of solution by using different starting solutions. Therefore, what is presented in this paper can be seen as the initial step of a bigger project, that we expect to pursue in the future.

Aside from the good model calibration we have reached, the most important advantages of the TRILEGAL code can be identified in: (1) the fairly complete database of stellar evolutionary tracks already implemented; (2) the use of an extended spectral library to simulate many different photometric systems, and their extinction coefficients, in a self-consistent way; (3) the modular and flexible structure of the code, that allows easy changes and additions to both input functions (SFR, AMR, IMF, etc.) and geometric parameters (the density of Galaxy components, Sun's position, pointing parameters, etc.).

With respect to other population synthesis codes commonly used to simulate the photometry of Galaxy fields (e.g. Ng et al. 1995; Vallenari et al. 2000; Castellani et al. 2002; Robin et al. 2003), TRILEGAL shares the advantage of being intrinsically self-consistent in the relative numbers of stars predicted to be in different evolutionary phases (including stellar remnants such as white dwarfs). In fact, for a given Galaxy geometry, stellar number ratios are univoquely determined by the choice of SFR, AMR and IMF, and are not tunable parameters. In TRILEGAL, this self-consistency of population synthesis codes is kept as a very stringent criteria, since there are explicit checks for the continuity of all stellar quantities (including core mass, envelope mass, and surface chemical composition when applicable) in the isochrone-construction routines that make part of the code.

As already mentioned, a main positive characteristic of TRILEGAL consists in the extreme flexibility in the way input libraries (evolutionary tracks, atmospheres) and functions 
(geometry, IMF, SFR, AMR of Galaxy components) can be changed, tested, and added to a database for future use. Improvements in the stellar evolutionary tracks, for instance those described in Marigo et al. (2003), have been inserted in TRILEGAL in almost no time. We have so far computed test models in at least 10 different photometric systems (including UBVRIJHK, Washington, HST-based instruments like WFPC2, NICMOS and ACS, the EIS photometric system, 2MASS and SDSS), and we are confident that virtually any broadband Vega, AB or ST magnitude system can be considered as well (cf. Girardi et al. 2002). Needless to say, before the present TRILEGAL calibration has been considered as acceptable, we have made wide use of its flexibility by testing many different IMFs, AMRs, SFRs, and density functions published by different authors. Such a flexibility is of fundamental importance for facing the huge amount of wide-field photometric data that is becoming available these days, and to take immediate advantage of the next generation of improved/expanded stellar evolutionary and atmospheric models, that are now being prepared by different groups around the world.

Therefore, the TRILEGAL code is ready to use for the variety of wide-angle surveys in the optical/infrared that will become available in the coming years and will provide constraints that will help us to pin down the structure of our Galaxy.

Acknowledgements. This publication makes use of data products from the Two Micron All Sky Survey, which is a joint project of the University of Massachusetts and the Infrared Processing and Analysis Center/California Institute of Technology, funded by the National Aeronautics and Space Administration and the National Science Foundation. We acknowledge the referee for the interesting remarks, which helped to improve this paper.

\section{References}

Allard, F., Hauschildt, P. H., Alexander, D. R., Tamanai, A., \& Ferguson, J. W. 2000, in From giant planets to cool stars, ed. C. A. Griffith \& M. S. Marley, ASP Conf. Ser., 212, 127

Arnouts, S., Vandame, B., Benoist, C., et al. 2001, A\&A, 379, 740

Bahcall, J. N. 1986, ARA\&A, 24, 577

Bahcall, J. N., \& Soneira, R. M., 1980, ApJS, 44, 73

Bahcall, J. N., \& Soneira, R. M., 1984, ApJS, 55, 67

Barmina, R., Girardi, L., \& Chiosi, C. 2002, A\&A, 385, 847

Benvenuto, O. G., \& Althaus, L. G. 1999, MNRAS, 303, 30

Bertelli, G., \& Nasi, E. 2001, AJ, 121, 1013

Bertelli, G., Bressan, A., Chiosi, C., Fagotto, F., \& Nasi, E. 1994, A\&AS 106, 275

Bessell, M. S., Castelli, F., \& Plez, B. 1998, A\&A, 333, 231

Binney, J., Gerhard, O., \& Spergel, D. 1997, MNRAS, 288, 365

Cardelli, J. A., Clayton, G. C., \& Mathis, J. S. 1989, ApJ, 345, 245

Carraro, G., Girardi, L., \& Marigo, P. 2002, MNRAS, 332, 705

Castellani, V., Cignoni, M., Degl'Innocenti, S., Petroni, S., \& Prada Moroni, P. G. 2002, MNRAS, 334, 69

Castelli, F., Gratton, R. G., \& Kurucz, R. L. 1997, A\&A, 318, 841

Chabrier, G. 2001, ApJ, 554, 1274

Chabrier, G., Baraffe, I., Allard, F., \& Hauschildt, P. H. 2000, ApJ, 542,464

Chen B., Stoughton C., Smith J. A., et al. 2001, ApJ, 553, 184

Cohen, M. 1995, ApJ, 444, 874
Cutri, R. M., Skrutskie, M. F., Van Dyk, S., et al. 2003, All-Sky Data Release Explanatory Supplement, http://www.ipac. caltech.edu/2mass/releases/allsky/ doc/explsup.html

de Vaucoulers, G. 1959, in Handbuch der Physik, ed. S. Flugge (Berlin: Springer-Verlag), 53, 311.

ESA, 1997, The Hipparcos and Tycho Catalogues

Finley, D. S., Koester, D., \& Basri, G. 1997, ApJ, 488, 375

Fluks, M. A., Plez, B., The, P. S., et al. 1994, A\&AS, 105, 311

Fuhrmann, K. 1998, A\&A, 338, 161

Gilmore, G. 1984, MNRAS, 207, 223

Gilmore, G., \& Reid, N. 1983, MNRAS, 202, 1025

Girardi, L. 1997, Ph.D. Thesis, Universidade Federal do Rio Grande do Sul, Porto Alegre, Brazil

Girardi, L. 1999, MNRAS, 308, 818

Girardi, L., \& Bertelli, G. 1998, MNRAS, 300, 533

Girardi, L., \& Salaris, M., 2001, MNRAS, 323, 109

Girardi, L., Bressan, A., Chiosi, C., Bertelli, G., \& Nasi, E. 1996, A\&AS, 117, 113

Girardi, L., Groenewegen, M. A. T., Weiss, A., \& Salaris, M. 1998, MNRAS, 301, 149

Girardi, L., Bressan, A., Bertelli, G., \& Chiosi, C. 2000, A\&AS, 141, 371

Girardi, L., Bertelli, G., Bressan, A., et al. 2002, A\&A, 391, 195

Girardi, L., Bertelli, G., Chiosi, C., \& Marigo, P. 2003, IAUS, 212 , 551

Girardi, L., Grebel, E. K., Odenkirchen, M., \& Chiosi, C. 2004, A\&A, 422, 205

Grebel, E. K., \& Roberts, W. J. 1995, A\&AS, 109, 293

Groenewegen M. A. T., Girardi L., Hatziminaoglou E., et al. 2002, A\&A, 392, 741 (Paper I)

Hall, P. B., Osmer, P. S., Green, R. F., Porter, A. C., \& Warren, S. J. 1996, ApJS, 104, 185

Hatziminaoglou, E., Groenewegen, M. A. T., da Costa, L., et al. 2002, A\&A, 384, 81

Haywood, M. 1994, A\&A, 282, 444

Henry, R. B. C., \& Worthey, G. 1999, PASP, 111, 919

Hernandez, X., Valls-Gabaud, D., \& Gilmore, G. 2000, MNRAS, 316, 605

Holmberg, J., \& Flynn, C. 2004, MNRAS, 352, 440

Homeier, D., Koester, D., Hagen, H.-J., et al. 1998, A\&A, 338, 563

Kroupa, P. 2001, MNRAS, 322, 231

Kurucz, R. L. 1993, in The Stellar Populations of Galaxies, ed. B. Barbuy, A. Renzini (Dordrecht, Kluwer), IAU Symp., 149, 225

Larson, R. B. 1986, MNRAS, 218, 409

Lyngå, G. 1982, A\&A, 109, 213

Marigo, P. 2002, A\&A, 387, 507

Marigo, P., Girardi, L., \& Chiosi, C. 2003, A\&A, 403, 225

Maíz-Apellániz, J. 2001, AJ, 121, 2737

Méndez, R. A., \& van Altena, W. F. 1996, AJ, 112, 655

Méndez, R. A., \& van Altena, W. F. 1998, A\&A, 330, 910

Ng, Y. K., Bertelli, G., Bressan, A., Chiosi, C., \& Lub, J. 1995, A\&A, 295, 655

Ng, Y. K., Bertelli, G., Chiosi, C., \& Bressan, A. 1997, A\&A, 324, 65

Ng, Y. K., Brogt, E., Chiosi, C., \& Bertelli, G. 2002, A\&A, 392, 1129

Ojha, D. K. 2001, MNRAS, 322, 426

Osmer, P. S., Kennefick, J. D., Hall, P. B., \& Green, R. F. 1998, ApJS, 119, 189

Parenago, P. P. 1945, Russian AJ, 22, 129

Perryman, M. A. C., Lindegren, L., Kovalevsky, J., et al. 1997, A\&A, 323, L49

Prandoni, I., Wichmann, R., da Costa L., et al. 1999, A\&A, 345, 448

Rana, N. C., \& Basu, S. 1992, A\&A, 265 ,499 
Reid, N., \& Majewski, S. R. 1993, ApJ, 409, 635

Renzini, A., \& da Costa, L. N. 1997, The Messenger, 87, 23

Robin, A. C., \& Crézé, M. 1986, A\&A, 157, 71

Robin, A. C., Reylé, C., \& Crézé, M. 2000, A\&A, 359, 103

Robin, A. C., Reylé, C., Derriére, S., \& Picaud, S. 2003, A\&A, 409, 523

Rocha-Pinto, H. J., Maciel, W. J., Scalo, J., \& Flynn, C. 2000, A\&A, 358,850

Ryan, S. G., \& Norris, J. E. 1991, AJ, 101, 1865

Salaris, M., \& Weiss, A. 1998, A\&A, 335, 943

Salaris, M., Chieffi, A., \& Straniero, O. 1993, ApJ, 414, 580

Salasnich, B., Girardi, L., Weiss, A., \& Chiosi, C. 2000, A\&A, 361, 1023

Salpeter, E. E. 1955, ApJ, 121, 161
Schlegel, D. J., Finkbeiner, D. P., \& Davis, M. 1998, ApJ, 500, 525

Schröder, K.-P., \& Pagel, B. E. J. 2003, MNRAS 343, 1231

Vallenari, A., Bertelli, G., \& Schmidtobreick, L. 2000, A\&A, 364, 925

Vandame, B., Olsen, L. F., Jorgensen, H. E., et al. 2001 [arXiv: astro-ph/0102370]

VandenBerg, D. A. 2000, ApJS, 129, 315

Vassiliadis, E., \& Wood, P. R. 1994, ApJS, 92, 125

York, D. G., Adelman, J., Anderson, J. E., Jr., et al. 2000, AJ, 120, 1579

Young, P. J. 1986, AJ, 81, 807

Zaggia, S., Hook, I., Mendez, R., et al. 1999, A\&AS, 137, 75

Zheng, Z., Flynn, C., Gould, A., Bahcall, J. N., \& Salim, S. 2001, ApJ, 555,393 


\section{Online Material}


L. Girardi et al.: Star counts, Online Material p 2

\section{Appendix A: Simulation of parallax errors in Hipparcos}

In this Appendix we describe how the parallax errors in the Hipparcos catalogue have been simulated. From the about 118000 objects in the Hipparcos catalogue the about 99000 objects have been selected that fulfill: "goodness-of-fit" flag (H30) less than 3, "percentage-of-rejected-data" flag (H29) less than 10, "number of components" flag (H58) of 1, that have a $V$-band magnitude and a parallax larger than 0.5 mas. Figure A.1 shows the distribution of errors $\sigma \pi$ for these data. From this dataset the following recipe was devised.

The median parallax error (in mas) is calculated from:

$\sigma_{\pi}^{\mathrm{med}}=0.914\left(\frac{V}{10}\right)^{6.1827}+0.735$

and the minimum parallax error (in mas) from:

$\sigma_{\pi}^{\min }=0.516\left(\frac{V}{10}\right)^{5.6010}+0.451$.

Then a random number is drawn from a Gaussian distribution with a mean of 1.0 and a sigma of 0.180 . This number is multiplied with $\sigma_{\pi}^{\text {med }}$. If this value is larger than $\sigma_{\pi}^{\min }$ then this is accepted as the parallax error $\sigma \pi$. If not, (a) new random number(s) is (are) drawn until this is fulfilled.

Once a star of magnitude $V$ and his error $\sigma \pi$ are simulated, the individual measurement error $\delta \pi$ is drawn from the Gaussian of dispersion $\sigma \pi$.

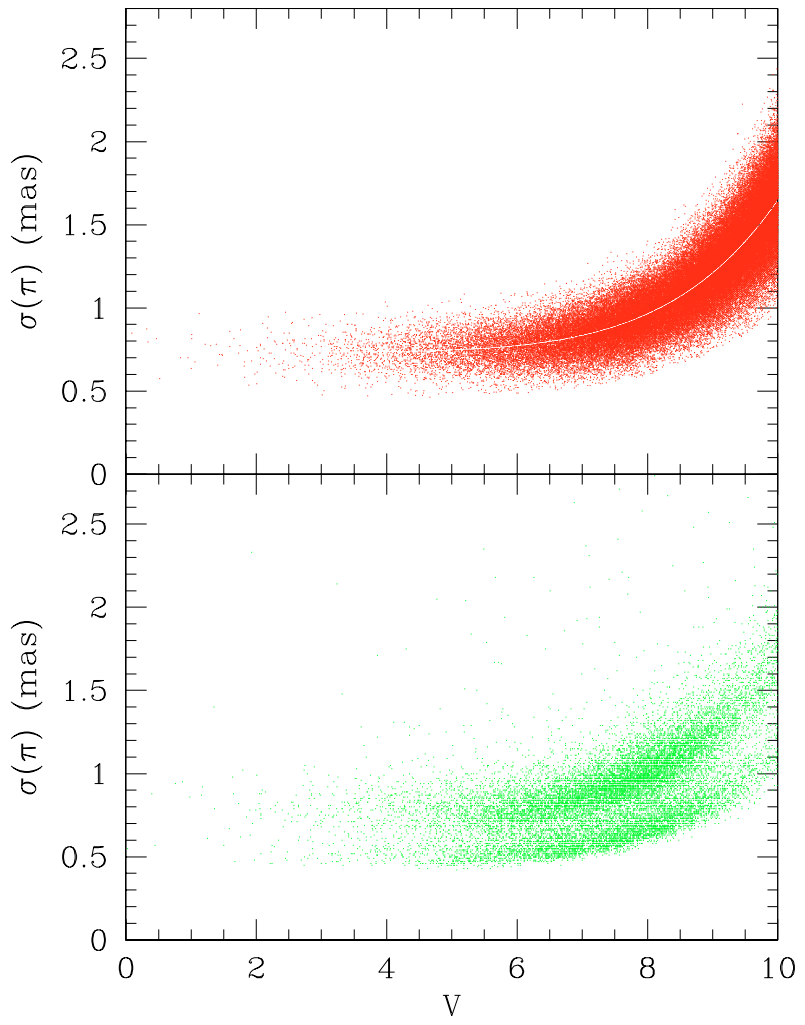

Fig. A.1. Errors in Hipparcos parallaxes. The data is in the lower panel, our simulations in the upper one. 